\title{
Models and Algorithms for the Next Generation of Glass Transition Studies
}

\author{
Andrea Ninarello, Ludovic Berthier, and Daniele Coslovich \\ Laboratoire Charles Coulomb, Université de Montpellier, CNRS, 34095 Montpellier, France \\ (Received 19 January 2017; revised manuscript received 28 April 2017; published 7 June 2017)
}

\begin{abstract}
Successful computer studies of glass-forming materials need to overcome both the natural tendency to structural ordering and the dramatic increase of relaxation times at low temperatures. We present a comprehensive analysis of eleven glass-forming models to demonstrate that both challenges can be efficiently tackled using carefully designed models of size polydisperse supercooled liquids together with an efficient Monte Carlo algorithm where translational particle displacements are complemented by swaps of particle pairs. We study a broad range of size polydispersities, using both discrete and continuous mixtures, and we systematically investigate the role of particle softness, attractivity, and nonadditivity of the interactions. Each system is characterized by its robustness against structural ordering and by the efficiency of the swap Monte Carlo algorithm. We show that the combined optimization of the potential's softness, polydispersity, and nonadditivity leads to novel computer models with excellent glass-forming ability. For such models, we achieve over 10 orders of magnitude gain in the equilibration time scale using the swap Monte Carlo algorithm, thus paving the way to computational studies of static and thermodynamic properties under experimental conditions. In addition, we provide microscopic insight into the performance of the swap algorithm, which should help optimize models and algorithms even further.
\end{abstract}

DOI: 10.1103/PhysRevX.7.021039

\section{INTRODUCTION}

Computer simulations play an increasingly important role in elucidating the nature of the glass transition because they allow particle-level resolution of any relevant static or dynamic observable [1]. While a similar spatial resolution can now be achieved in experiments performed with colloids [2], less direct microscopic information is available from experimental studies of molecular liquids [3]. Regarding time scales, however, colloidal experiments and computer simulations cover, at best, the first 4-5 decades of the dynamic slowing down of systems approaching a glass transition [4], whereas 12-13 orders of magnitude of glassy slowdown can be analyzed in molecular liquids [5]. Therefore, the exquisite level of detail gained from simulations in the description of the onset of slow dynamics concerns a dynamical regime that is separated from experiments on molecular glasses by about 8 orders of magnitude. The dichotomy between accessible length scales and time scales is a major challenge for glass transition studies [1,6,7].

There are several promising experimental advances that could improve either the dynamic range of colloidal experiments [8] or the spatial resolution in molecular

Published by the American Physical Society under the terms of the Creative Commons Attribution 4.0 International license. Further distribution of this work must maintain attribution to the author(s) and the published article's title, journal citation, and DOI.
Subject Areas: Chemical Physics, Soft Matter,

Statistical Physics supercooled liquids [9]. In addition, new protocols to prepare molecular glasses corresponding to even larger relaxation times are being developed [10]. On the simulation front, the situation appears challenging, as the increase in the time window accessible to computer simulations has been rather slow, amounting to a gain of about 3 orders of magnitude over the last 30 years $[4,11,12]$, and this is mostly due to improvements in computer hardware. A rough extrapolation of this trend would pessimistically suggest that it could take another 100 years for simulations to close the gap with experimentally relevant thermodynamic conditions. The recent advent of graphic processing units and accelerators in the high-performance computing arena suggests that progress could be made at a faster pace if novel technologies become available. Exploiting them in the context of molecular simulations [13-17] nonetheless requires a substantial investment in code development and low-level optimization.

The above summary suggests that it is desirable to develop alternative strategies that do not simply rely on the brute-force increase of computing power. A possible path is to take advantage of the flexibility offered by simulations and implement algorithms that simulate equilibrium material properties more efficiently [18]. Several such strategies have already been explored. A first line of research concerns the development of collective particle displacements to improve sampling efficiency [19-21]. This approach follows the method employed to study phase transitions in spin systems [22,23]. For instance, the event-chain Monte Carlo algorithm has proved useful in 
the study of two-dimensional melting [21], but its gain in efficiency for the three-dimensional dense fluids considered here is, at most, a factor of 40 [24], which remains insufficient to close the gap with experiments. A crucial aspect for the efficiency of this approach is the choice of the correct type of collective move, which still requires some a priori knowledge of the relaxation path used by the system [25]. However, this information remains to be understood in fragile glass-forming materials.

A different simulation strategy is the replica-exchange technique, where simulations of the same system are conducted in parallel over a range of state points, and infrequent exchanges between neighboring state points are performed [26-29]. The idea is that navigating through different state points would facilitate the crossing of large barriers in a complex free-energy landscape, and indeed, the technique was first developed to study spin glasses [26]. In dense fluids, the reported speedup is again of about 2 orders of magnitude [28,29], with the additional drawback that the replica-exchange technique scales very poorly with the number of particles and loses most of its efficiency for system sizes of thousands of particles, which are typically used in studies of the bulk glass transition [29]. Therefore, replica exchange works best for studies of equilibrium phase transitions in small systems, as confirmed in a series of recent studies [30-33]. Different algorithms such as Wang-Landau sampling [34] and population dynamics [35] have also been employed in the context of glass studies.

The swap Monte Carlo algorithm is another long-standing simulation technique that has been used in computer studies of the glass transition. The algorithm was first applied to the study of the equation of state of a nonadditive hard sphere system [36] and later rediscovered in the context of the glass transition of a binary mixture of soft spheres [37]. The swap algorithm has since been mostly used in the glass context, for both binary mixtures [38-41] and for continuously polydisperse systems [42-44]. For the binary mixture of Ref. [45], the reported speedup in terms of equilibration times is a factor of 180 , independent of temperature [46]. The glass-forming ability of this model is, however, poor because of the appearance of ordered phases $[37,47,48]$. Little quantitative information is available concerning the efficiency of the swap algorithm for continuously polydisperse soft $[43,44,47]$ and hard spheres [49]. In an effort to improve the stability of discrete mixtures, Gutierrez et al. recently introduced a ternary mixture of soft spheres to study the increase of a static length scale [50]. Very low temperatures were studied, and

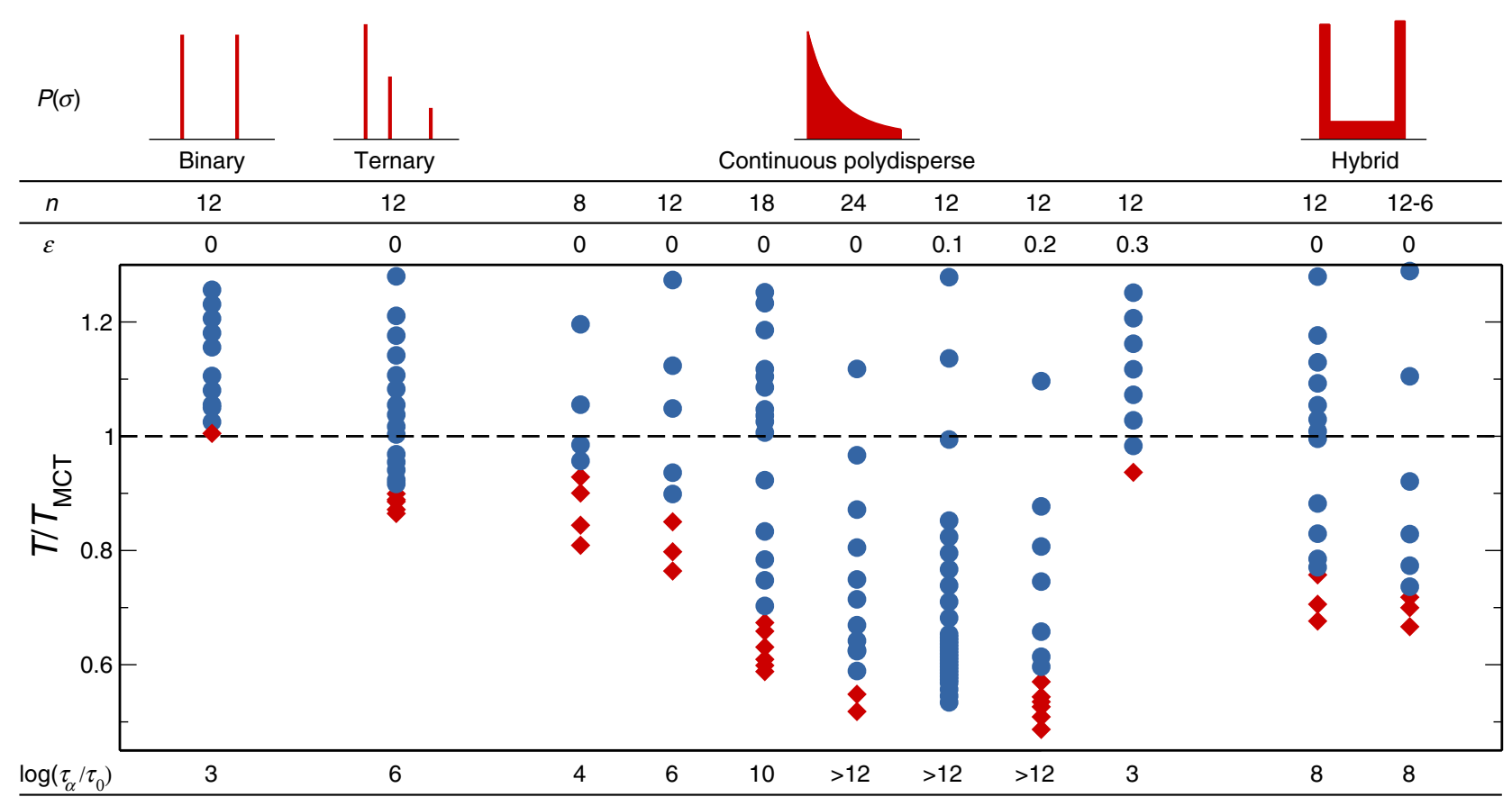

FIG. 1. Summary of the results obtained for the eleven models studied in this work. The top row shows the particle size distribution for each model; the next two rows specify the pair potential and its additivity. Below, we use a temperature axis rescaled by the location of the mode-coupling crossover, where blue points indicate equilibrium disordered fluid configurations and red diamonds indicate instability towards crystalline or demixed states. The bottom row indicates the estimated range of equilibrium relaxation times $\tau_{\alpha}$ that can be studied in stable equilibrium conditions for each model, using $\tau_{0}$ as the relaxation time at the onset temperature [53]. We have constructed several models that remain stable and can be equilibrated deep in the temperature regime $T / T_{\text {MCT }}<1$, which conventional simulation studies are unable to penetrate, three of which allow us to reach temperatures below the experimental glass transition, conventionally defined as $\tau_{\alpha} / \tau_{0}=10^{12}$. 
a claim of a 10-decade efficiency gain was made. We demonstrate below that changing from a binary to a ternary mixture indeed improves the thermodynamic stability, but the claims made in Ref. [50] do not resist our detailed analysis of the structure and thermalization of the model. We demonstrate that the efficiency gain for this model is much more modest, and the accessible dynamical window is increased by about $2-3$ orders of magnitude.

The aim of our work is to bring the swap algorithm to a whole new level of performance. We present a systematic study of glass-forming ability and thermalization efficiency over a broad range of glass-forming models, varying the particle size distribution and the nature of the pair interactions while optimizing the swap Monte Carlo algorithm. Our main result, summarized in Fig. 1, is the discovery that particular combinations of parameters yield both excellent glass-forming ability and a dramatic decrease of the computer time needed to obtain thermalized configurations at low temperatures. This insight has already led to some new results on related phenomena, such as jamming [51] and the Gardner transition [52].

As shown in Fig. 1, we systematically change the size distribution, using a variety of discrete and continuous mixtures, we vary the softness of the pair repulsion and its additivity, and we add attractive forces. For each case, we determine both the temperature regime where the model is structurally unstable (shown with red symbols) and the temperature regime where the disordered fluid state is stable at equilibrium (shown with blue symbols). The vertical axis represents the temperature $T$, scaled by the location of the corresponding mode-coupling crossover, $T_{\text {MCT }}$. Although somewhat arbitrary, this rescaling demonstrates the efficiency of the thermalization because conventional computer simulations typically fail to reach equilibrium in the regime $T / T_{\mathrm{MCT}}<1$. Despite the differences between systems, several of them can be thermalized in the supercooled liquid state at significantly lower temperatures than ordinary simulations. We demonstrate that this temperature regime corresponds, for some of these models, to a range of relaxation times of more than 12 decades, which implies that we can access, in equilibrium, a temperature regime that is even lower than the experimental glass transition temperature $T_{g}$. We show that this corresponds to a speed-up of the thermalization of about 10 orders of magnitude at $T_{g}$.

The two key factors enabling such progress are the use of an appropriate size polydispersity to prevent both crystallization (when polydispersity is too small) and phase separation (when it gets too large), and a particle size distribution that allows for a large acceptance rate for particle swaps, in turn leading to a fast thermalization and equilibrium sampling of phase space.

The outline of the article is as follows. Section II is dedicated to the simulation strategy and technicalities. Results for two families of systems (mixtures and continuous polydisperse systems) are reported and discussed, respectively, in Secs. III and IV. We give a physical insight on swap dynamical relaxation and heterogeneities in Sec. V. Section VI deals with the introduction of a model designed to maximize the algorithm efficiency. Finally, Sec. VII presents our conclusions and offers further perspectives for future work.

\section{DETAILS OF THE SIMULATIONS}

\section{A. Algorithm, interactions, and size distributions}

We simulate systems of $N$ particles in a cubic box of side $L$ with periodic boundary conditions [54]. Throughout the paper, we compare results obtained from two kinds of simulation methods: standard Monte Carlo simulations in the canonical ensemble [55] and swap Monte Carlo simulations [36,37]. Both simulation algorithms involve the same displacement moves, in which we pick up one particle at random and attempt to translate it by a displacement vector randomly drawn in a cube of linear size $\delta l$. The move is accepted using the Metropolis acceptance rule, which ensures that detailed balance is obeyed at each temperature $T$. For each model, the typical jump length $\delta l$ is fixed to a fraction of the average particle diameter, which results in an acceptance rate ranging typically from about $60 \%$ at high temperatures to $30 \%$ at low temperatures. This approach to simulating glass formers has been validated by direct comparison with molecular-dynamics results for the specific case of a binary mixture [56].

In addition to displacement moves, during a swap Monte Carlo simulation, we also attempt to exchange the diameters of two randomly chosen particles. The diameter exchange is again accepted based on the Metropolis criterion. At every Monte Carlo step, such a "swap move" is attempted with probability $p$. We emphasize that swap moves preserve detailed balance and thus guarantee an equilibrium sampling of phase space [18]. In other words, despite the "nonphysical" nature of the swap moves (in an experiment, particles would not exchange their diameters spontaneously), the swap Monte Carlo dynamics enables a proper sampling of the equilibrium thermodynamic properties of the model. In previous implementations of the swap Monte Carlo dynamics, particle swaps were described as particles exchanging their positions, instead of their diameters [37]. Both descriptions are, of course, fully equivalent, but our choice offers the advantage that single-particle dynamics can be followed in time because particles do not make arbitrarily large jumps during the swap moves. Standard time correlation functions based on particle displacements can thus be measured in swap and ordinary Monte Carlo simulations in the exact same way. Dynamic measurements are a crucial tool to assess the thermalization of our swap simulations, just as they are for standard simulations of supercooled liquids. One Monte Carlo sweep is then defined as $N$ consecutive 
attempts to either displace or swap particle diameters, and one such sweep will represent our time unit in the following.

In this work, we study three different classes of systems, with particle size distributions as sketched in Fig. 1. They are either discrete or continuous mixtures. Discrete mixtures are characterized by a particle size distribution $P(\sigma)$ of the form

$$
P(\sigma)=\sum_{\alpha=1}^{m} x_{\alpha} \delta\left(\sigma-\sigma_{\alpha}\right)
$$

where $m$ is the total number of components, $x_{\alpha}$ indicates the fractional composition of each species, and $\sigma_{\alpha}$ is the diameter of species $\alpha$. Within the class of continuously polydisperse systems, we focus on a specific kind of size dispersity, which scales as the inverse of the occupied volume:

$$
P(\sigma)=\frac{A}{\sigma^{3}}, \quad \sigma \in\left[\sigma_{\min }, \sigma_{\max }\right],
$$

where $A$ is a normalizing constant and $\sigma_{\min }$ and $\sigma_{\max }$ are the minimum and the maximum diameter values, respectively. This functional form ensures that the volume fraction occupied by particles within a given size bin is constant. Such a scaling property has been shown to enhance the glass-forming ability in discrete mixtures [57], but we have not tested this hypothesis in great detail for the present systems.

Finally, we introduce a second type of continuous particle size distribution, which combines the salient features of both discrete and continuous mixtures. Thus, we call this a "hybrid" distribution; see Fig. 1. Mathematically, the distribution reads

$$
P(\sigma)=\sum_{\alpha=1}^{m} x_{\alpha} \theta\left(b_{\alpha}-\left|\sigma-\sigma_{\alpha}\right|\right)
$$

where $\theta(x)$ is the Heaviside function and $x_{\alpha}$ is defined as before. In this approach, each component of the "mixture" is characterized by a flat particle size distribution of width $b_{\alpha}$. The goal is to construct models that combine advantages of both discrete mixtures, which are typically good glass formers, and continuous distributions, for which swap dynamics is very efficient.

We quantify the degree of polydispersity of a system by the normalized root-mean-square deviation

$$
\delta=\frac{\sqrt{\left\langle\sigma^{2}\right\rangle-\langle\sigma\rangle^{2}}}{\langle\sigma\rangle}
$$

where the brackets indicate an average of the particle size distribution. In the following, we use $\langle\sigma\rangle=\int P(\sigma) \sigma d \sigma$ as the unit length for each model studied.
We model the interactions between two particles $i$ and $j$ via a soft repulsive pair potential of the type

$$
v\left(r_{i j}\right)=\left(\frac{\sigma_{i j}}{r_{i j}}\right)^{n}+F\left(r_{i j}\right),
$$

where $n$ is an exponent controlling the softness of the repulsive potential and $F\left(r_{i j}\right)$ is a function that smooths the potential at the cutoff distance $r_{\text {cut }}$, beyond which the potential is set to zero. Unless otherwise specified, we use [50]

$$
F\left(r_{i j}\right)=c_{0}+c_{2}\left(\frac{r_{i j}}{\sigma_{i j}}\right)^{2}+c_{4}\left(\frac{r_{i j}}{\sigma_{i j}}\right)^{4} .
$$

The coefficients $c_{0}, c_{2}$, and $c_{4}$ ensure the continuity of the potential up to the second derivative at the cutoff distance $r_{\text {cut }}=1.25 \sigma_{i j}$. Additionally, we studied a polydisperse model where particles interact with the Lennard-Jones potential

$$
v\left(r_{i j}\right)=\left(\frac{\sigma_{i j}}{r_{i j}}\right)^{12}-\left(\frac{\sigma_{i j}}{r_{i j}}\right)^{6}+c_{L J}
$$

for which we simply cut off and shift the pair potential at the cutoff distance $r_{\text {cut }}=2.5 \sigma_{i j}$.

Finally, to ensure a high structural stability in our models, we introduce a generalized nonadditive interaction rule for the cross diameters $\sigma_{i j}$ in the pair interaction, which reads

$$
\sigma_{i j}=\frac{\sigma_{i}+\sigma_{j}}{2}\left(1-\epsilon\left|\sigma_{i}-\sigma_{j}\right|\right) .
$$

Systems characterized by $\epsilon=0$ and $\epsilon \neq 0$ will be referred to as additive and nonadditive systems, respectively. Nonadditivity is another ingredient that has been widely used to enhance glass-forming ability in simple binary models [58], and it is a consequence of the band structure of the electronic density of states in metallic alloys [59]. Physically, the nonadditive rule in Eq. (8) implies that particles with identical diameters interact as before but that small and large particles can have a larger overlap than for additive systems.

\section{B. Physical observables}

In this section, we introduce the basic observables used to characterize the structure and dynamics of the models studied. We use them to monitor the equilibration and the stability of the fluids under supercooled conditions and to quantify and compare the degree of thermalization achieved by both standard and swap simulations. 
We systematically compute the structure factor [60],

$$
S(k)=\frac{1}{N}\left\langle\rho_{\mathbf{k}} \rho_{-\mathbf{k}}\right\rangle,
$$

where $\rho_{\mathbf{k}}$ is the Fourier transform of the microscopic density at wave vector $\mathbf{k}$. The behavior of $S(k)$ at small wave-number provides information on possible long-range density fluctuations and will be checked to identify signals of instability of the homogeneous fluid. Since we deal with size-disperse systems, we compute partial structure factors associated with each subpopulation. In the case of continuously polydisperse systems, we group particles of comparable size into families labeled by an index $\alpha$, for which we compute the partial structure factor $S_{\alpha \alpha}(k)$. A strong increase of $S_{\alpha \alpha}(k)$ at small $k$ values is associated with phase separation or demixing, and we have monitored this quantity systematically in our models.

Besides particle demixing, the main instability overcome is, of course, crystallization. To detect the presence of crystalline local order, we measure the sixfold bondorientational order parameter [61]

$Q_{6}=\left\langle\frac{1}{N} \sum_{i} \sqrt{\frac{4 \pi}{13} \sum_{m=-6}^{6}\left|\frac{1}{N_{b}(i)} \sum_{j=1}^{N_{b}(i)} Y_{6 m}\left(r_{i j}\right)\right|^{2}}\right\rangle$,

where $Y_{6 m}\left(r_{i j}\right)$ are spherical harmonics. The sum over $1<$ $j<N_{b}(i)$ runs over the neighbors of particle $i$ in a sphere of radius corresponding to the minimum of the distribution function of rescaled interparticle distances, $g\left(r_{i j} / \sigma_{i j}\right)$. We inspect the time and temperature variation of $Q_{6}$, as well as that of the potential energy $e$, to check whether the systems are stable against crystallization.

We provide a systematic characterization of both selfdynamics and collective dynamics of the models. This enables us to quantify the degree to which swap simulations enhance thermalization compared to standard Monte Carlo dynamics. We note that while standard Monte Carlo dynamics [56] can be used to mimic overdamped Brownian dynamics, as appropriate for a colloidal suspension [4], the microscopic dynamics of swap simulations is not physical. We emphasize, however, that the particles' trajectories remain well defined because the swap moves only exchange the particle diameters not their positions. Thus, even though the microscopic dynamics is nonphysical, time-dependent correlation functions still quantify the time scale over which individual particles diffuse (for selfcorrelation functions) and over which the density fluctuations relax (for collective correlations). Time correlation functions will be used in the following to determine whether the system has been efficiently thermalized at a given state point.

We characterize the single-particle dynamics through the self-part of the intermediate scattering function

$$
F_{s}(k, t)=\left\langle f_{s}(k, t)\right\rangle=\left\langle\frac{1}{N} \sum_{j} e^{i \mathbf{k} \cdot\left[\mathbf{r}_{j}(t)-\mathbf{r}_{j}(0)\right]}\right\rangle,
$$

where the wave number $k$ corresponds to the first peak of the total structure factor $S(k)$. Notice that since the particle diameter changes during the course of the simulations, the sum in Eq. (11) runs over all particles, the distinction between large and small particles being immaterial. The structural relaxation time $\tau_{\alpha}$ is then defined as the value at which $F_{s}\left(k, \tau_{\alpha}\right)=e^{-1}$, following common practice. We use the relaxation time $\tau_{\alpha}$ measured for standard Monte Carlo simulations to locate the mode-coupling crossover at $T=T_{\mathrm{MCT}}$, which we take as a relevant temperature scale for computer simulations. In order to obtain $T_{\mathrm{MCT}}$, we fit the standard dynamics (without swap) in the interval $\tau_{0}<\tau_{\alpha}<10^{3} \tau_{0}$ with a power-law divergence [62],

$$
\tau_{\alpha} \propto\left(T-T_{\mathrm{MCT}}\right)^{-\gamma} .
$$

When discussing the dynamics of our models, we also use other functional forms to describe the temperature evolution of the relaxation time. A well-known functional form is the Vogel-Fulcher-Tamman (VFT) law [1],

$$
\tau_{\alpha}=\tau_{\infty} \exp \left(\frac{A}{T-T_{0}}\right)
$$

where $\tau_{\infty}, A$, and $T_{0}$ are fitting parameters. Because this functional form describes a dynamic singularity at a finite temperature $T=T_{0}$, it produces a very steep temperature dependence. A less pronounced temperature dependence is obtained with the parabolic law [63],

$$
\tau_{\alpha}=\tau_{\infty}^{\prime} \exp \left[A^{\prime}\left(\frac{1}{T}-\frac{1}{T_{1}}\right)^{2}\right],
$$

where $\tau_{\infty}^{\prime}, A^{\prime}$, and $T_{1}$ are again free parameters. Notice that no dynamic singularity is predicted from Eq. (14) since $T_{1}$ captures the onset of slow dynamics and not the divergence of the relaxation time at low temperatures. A final form that we use is the Arrhenius law,

$$
\tau_{\alpha}=\tau_{\infty}^{\prime \prime} \exp \left(\frac{A^{\prime \prime}}{T}\right)
$$

with $\tau_{\infty}^{\prime \prime}$ and $A^{\prime \prime}$ two fitting parameters.

Using these functional forms will be useful below to estimate the range of relaxation times that swap dynamics allows us to access. Our analysis shows that the VFT law presumably overestimates the growth of the relaxation time, whereas the Arrhenius law underestimates it, the parabolic law falling somewhere in between. Thus, the combination of all three fitting functions provides an 
estimate of the actual physical behavior and a sensible confidence interval in low-temperature extrapolations.

The relaxation of collective density fluctuations is measured via the time-dependent overlap function

$$
F_{o}(t)=\left\langle\frac{1}{N} \sum_{i, j} \theta\left(a-\left|\mathbf{r}_{i}(t)-\mathbf{r}_{j}(0)\right|\right)\right\rangle,
$$

using a cutoff distance $a=0.3$. This quantity provides similar information as the coherent intermediate scattering function at wave vector $k=2 \pi / a$, but it is computationally more advantageous because it presents smaller statistical fluctuations. From this function, we define a relaxation time $\tau_{o}$ for the decorrelation of collective density fluctuations, such that $F_{o}\left(\tau_{o}\right)=e^{-1}$.

For selected models, we computed a number of additional static and dynamical observables with the aim of understanding microscopic processes taking place during the swap Monte Carlo simulations. These more specific observables are described later in Sec. V.

\section{Efficiency of swap moves}

Because the swap Monte Carlo move is conceptually very simple, there are very few parameters that can be adjusted to optimize its efficiency. We discuss how to achieve maximal efficiency in the present section.

The extent to which swap moves accelerate the sampling of configuration space during a Monte Carlo simulation must depend on the frequency used to attempt such moves, which is given by the probability $p$. There are two obvious limiting cases. For $p=0$, one recovers the dynamics of a standard Monte Carlo simulation without swap moves. For $p=1$, instead, only swap moves are attempted; the particle positions are never updated, so by construction, structural relaxation cannot take place. The optimal choice for $p$ is thus the one that minimizes the structural relaxation time $\tau_{\alpha}$ of the system with respect to $p$.

We illustrate the optimization procedure for a continuously polydisperse particle system interacting via a soft repulsive potential as in Eq. (5) with $n=12$, with a nonadditivity $\epsilon=0.2$. This model is further discussed in Sec. IV C. The general trend found for this model is representative of the three classes of systems we investigated and is shown in Fig. 2, where we report the structural relaxation time of the system versus $p$ at a constant temperature, $T=0.101$. We normalized the relaxation time by the corresponding value in the absence of swap moves at $p=0$. At this particular temperature, we observe that the structural relaxation time becomes almost 3 orders of magnitude faster compared to standard dynamics already for very small values of $p$, i.e., $p$ of the order of a few percent. We observe a relatively broad minimum around $p \approx 0.2$ before $\tau_{\alpha}$ starts to grow again and diverges for $p=1$ as $\tau_{\alpha} \sim(1-p)^{-1}$, when particles stop diffusing

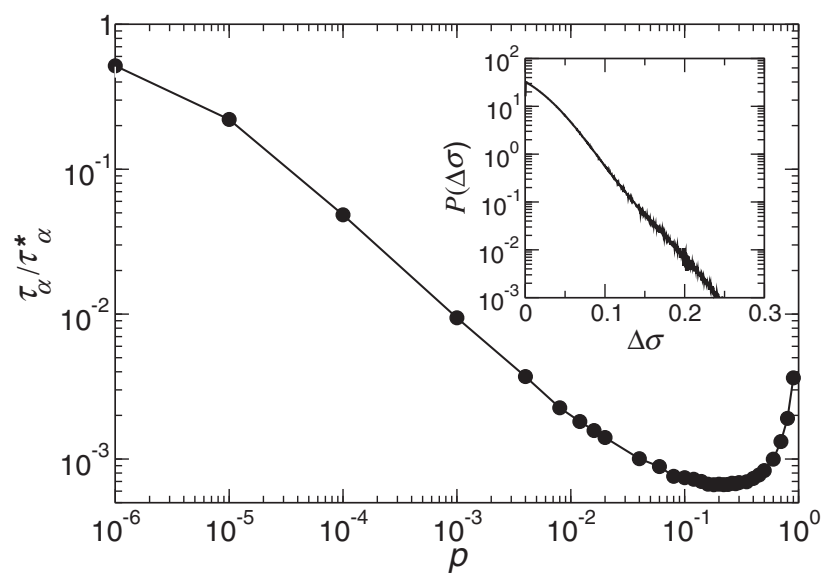

FIG. 2. The relaxation time $\tau_{\alpha}$ as a function of the swap attempt probability $p$, normalized by $\tau_{\alpha}^{*}$, its value for standard Monte Carlo simulations when $p=0$. A broad minimum indicates that $p \approx 0.20$ optimizes the efficiency of the swap Monte Carlo algorithm. The inset shows the probability distribution of swap acceptance as a function of the diameter difference $\Delta \sigma=\left|\sigma_{1}-\sigma_{2}\right|$ between the particles for which the swap move is attempted. The system is a soft repulsive system of nonadditive particles studied in Sec. IV C, with $\epsilon=0.2$ and $T=0.101$.

for the trivial reason mentioned above. From such a graph, we deduce that $p \approx 0.2$ is the optimal value for the probability to perform swap moves. We find that this value is fairly robust when temperature is changed or across different models, which presumably stems from the fact that the minimum reported in Fig. 2 is relatively flat. Another remarkable feature of this figure is the very steep decrease of $\tau_{\alpha}$ observed for even very small values of $p$, suggesting that even a fairly small amount of swap moves are in fact sufficient to enormously facilitate the structural relaxation of the system.

Efficiency considerations should also take into account the CPU time needed to perform a swap move as opposed to a standard displacement. An attempt to swap diameters entails the computation of the local energy variation between the new and the old configurations for two particles, which is twice what is needed for an ordinary displacement move involving only one particle. However, we found that the optimum value for $p$ barely changes even when taking this additional effect into account. In terms of CPU time, one MC sweep with $p=0.2$ takes only $20 \%$ longer on average than a standard sweep with $p=0$. This should be contrasted with the orders of magnitude of gain achieved in terms of structural relaxation time.

Another major advantage of the swap Monte Carlo algorithm is that both its implementation and its efficiency are insensitive to the number of particles in the system, $N$. This contrasts strongly with the replica exchange method, which scales very poorly with $N$ [26-29].

In general, the acceptance ratio of the Monte Carlo moves decreases upon lowering temperature or increasing 
the density. Similarly, the acceptance ratio of swap moves decreases when the size difference $\Delta \sigma=\left|\sigma_{1}-\sigma_{2}\right|$ of the two selected particles increases because a large particle will not easily fit into the hole occupied by a small one. As will be clear in the following, the efficiency of swap moves is highest in continuously polydisperse systems, where the diameter difference between any two particles can be arbitrarily small. In these systems, it is pertinent to avoid attempting exchanges when $\Delta \sigma$ is too large because the swap move is then essentially always rejected [47]. This point is illustrated in the inset of Fig. 2, where we show $P(\Delta \sigma)$, the probability distribution of acceptance rates for swap moves between pairs of particles with a size difference $\Delta \sigma$ for the same parameters as in the main frame of the figure. We notice that the acceptance rate decays exponentially fast with $\Delta \sigma$ and becomes vanishingly small when $\Delta \sigma \gtrsim 0.25$. Following Ref. [47], we therefore disregard swaps between particles with a diameter difference larger than a certain cutoff. Here, we choose $\Delta \sigma_{\max }=0.2$, which we found to be a reasonable trade-off. We implement this threshold value in a way that preserves detailed balance. In practice, we always choose two particles at random, but we directly reject the swap without evaluating any energy difference if $\Delta \sigma$ exceeds the chosen cutoff value.

\section{Equilibration and metastability}

Simulations of glass-forming liquids must be long enough to ensure equilibrium sampling of the observables of interest and yet short enough to avoid crystallization or more complex forms of structural ordering. Simple models such as binary mixtures or weakly polydisperse systems have been shown to crystallize over sufficiently long times [43,44,47,64-66]. Computer simulations of glass-forming materials thus always represent a narrow compromise between those two limits, which both need to be addressed carefully.

These issues become particularly severe when employing enhanced sampling algorithms, such as swap Monte Carlo moves that are precisely constructed to promote a more efficient exploration of phase space. For instance, crystallization of two-component mixtures of repulsive spheres has been reported in swap Monte Carlo simulations [47,48,67]. The ground-state of polydisperse repulsive particles was studied both with swap Monte Carlo simulations [43] and in the semi-grand canonical ensemble $[64,65]$. These studies found that for sufficiently high polydispersity, the stable structure is a fractionated crystal, where the system presents multiple crystals, each involving a fraction of the system's overall particle size distribution. However, as noticed in Ref. [64], the free energy cost of forming an interface between distinct phases is generally high, and crystallization may be difficult to observe in practice.

The general conclusion to be drawn from these earlier works is that a model considered as a good glass-forming system when studied using conventional simulation techniques may turn out to be a very poor model when using an enhanced simulation technique that is able to probe a much wider range of temperatures. Indeed, we find that many previously studied types of glass-forming models do not withstand basic stability criteria when the swap technique is applied, forcing us to develop novel numerical models in addition to the optimization of the swap Monte Carlo method.

To make a consistent comparison of the glass-forming ability of the studied models in standard and swap simulations, we follow a rigorous and identical equilibration protocol for all our models. First, we obtain static and dynamical properties of the system by means of standard Metropolis Monte Carlo simulations in the NVT ensemble [55]. From these simulations, we extract the average potential energy value, the structure factors, and the structural relaxation times. For each model, we determine $T_{\text {MCT }}$ using Eq. (12), which will serve as a reference temperature scale to compare the degree of supercooling across different models. This is not an ideal choice, but it offers the advantage that extrapolation of the relaxation times to low temperatures is not needed.

Swap Monte Carlo simulations start from a configuration equilibrated at the onset temperature $T_{o}$ [53], followed by an instantaneous quench to the target temperature $T$. The following criteria are used to determine whether the system has reached equilibrium at $T$. First, we monitor the potential energy $e$ per particle. We inspect both its instantaneous value as a function of time, $e(t, T)$, to detect aging, and its time average as a function of temperature, $\langle e\rangle(T)$, to detect possible discontinuities or change of slope in the equation of state of the liquid. Second, we ensure that the total mean-squared displacement $\Delta r^{2}(t)=$ $\left\langle 1 / N \sum_{i}\left[\mathbf{r}_{i}(t)-\mathbf{r}_{i}(0)\right]^{2}\right\rangle$ has reached a value at least larger than 6 . This specific value is relatively immaterial as this criterion only conveniently allows us to distinguish state points where particle displacements are large over the numerical time window, from those where particle dynamics is essentially arrested. Finally, we look at the selfincoherent scattering function. For this quantity, we check, within statistical fluctuations, both the absence of aging and the complete decorrelation to zero at long times. Once equilibration has been reached, we perform a first set of simulations to obtain a rough estimate of the structural relaxation time $\tau_{\alpha}$ in the presence of swap moves. After this is done, the system is simulated over a total of $200 \tau_{\alpha}$ to measure static and dynamic properties over a sufficiently wide time window. Notice that this thermalization procedure is rather demanding, and thermalization thus requires that we are able to perform simulations over a time window which is 2 orders of magnitude longer than the structural relaxation time. We emphasize that such a procedure is not specific to the presence of the swap moves. We think that simulations of supercooled liquid should 
follow similar strict rules to claim that equilibration, as well as a proper sampling of phase space, has been achieved.

Almost every system we simulated eventually displays some form of structural instability at sufficiently low temperatures, such as nucleation of an ordered phase or long-wavelength density fluctuations and demixing. These instabilities are detected via the observables introduced in the previous section. To decide whether a given state point remains in a metastable disordered fluid state, we use the following criterion. We perform five independent simulations along the lines described above. If at least one among the five samples shows an instability over a time window of $200 \tau_{\alpha}$, then we classify this state point as unstable and the system is a poor glass former at this temperature. The precise meaning of "instability" is system dependent and will be specified in each studied case in Secs. III and IV. This criterion is rather strict, as there could still be room for achieving thermalization and performing equilibrium sampling while avoiding structural instability, but this is dangerous as fluctuations related to ordering could interfere with the physics of the metastable disordered state. In addition, we find that when a state point is deemed unstable using our criterion, then lower temperatures are also unstable, and the instability rapidly becomes so severe that further studies cannot be safely performed. Thus, changing the details of our criterion would simply shift the range of "metastable" temperatures by a very small amount, and our conclusions would not be affected.

We first directly compare the thermalization efficiency of standard and swap simulations in Fig. 3. Starting from a high-temperature configuration, we progressively quench the system down to zero temperature using a constant cooling rate $\gamma=d T / d t$, with values changing

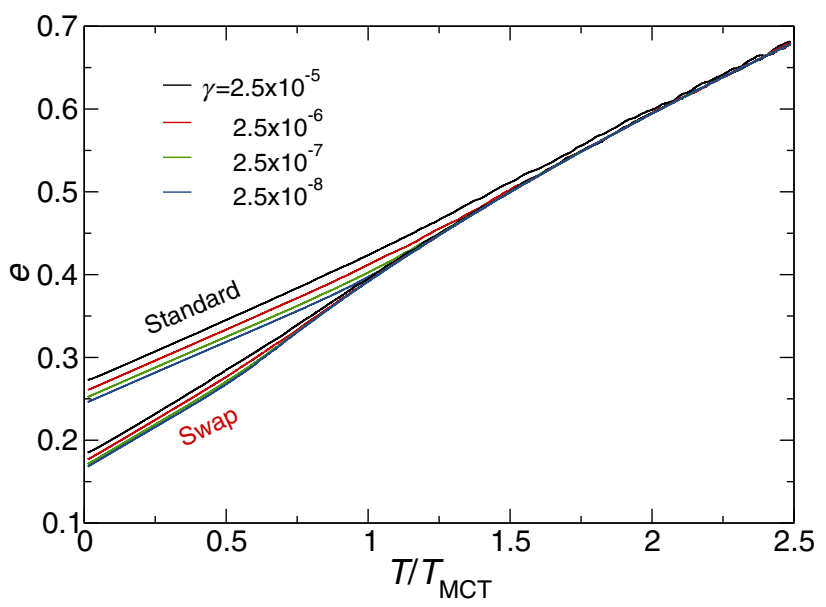

FIG. 3. Potential energy per particle in simulations with different cooling rates $\gamma$ using both standard and swap Monte Carlo dynamics. Both dynamics produce consistent results at high temperatures, but the swap dynamics remains at equilibrium down to much lower temperatures than the standard one. The system is the soft repulsive system of nonadditive particles studied in Sec. IV C, with $\epsilon=0.2$. logarithmically over a broad interval $\left(2.5 \times 10^{-5}\right.$, $\left.2.5 \times 10^{-9}\right)$. We further average our results using ten independent initial configurations. For standard Monte Carlo simulations, we retrieve the expected behavior where departure from the equilibrium equation state arises at lower temperatures for a lower cooling rate, as signaled by a rate dependence of the energy. Using swap simulations, we obtain the very same equation of state at equilibrium and a similar rate-dependent behavior at low temperatures. The major difference between the two sets of simulations is that swap simulations clearly fall out of equilibrium at considerably lower temperatures than ordinary Monte Carlo simulations. The agreement of the two sets of curves when they both probe equilibrium is an
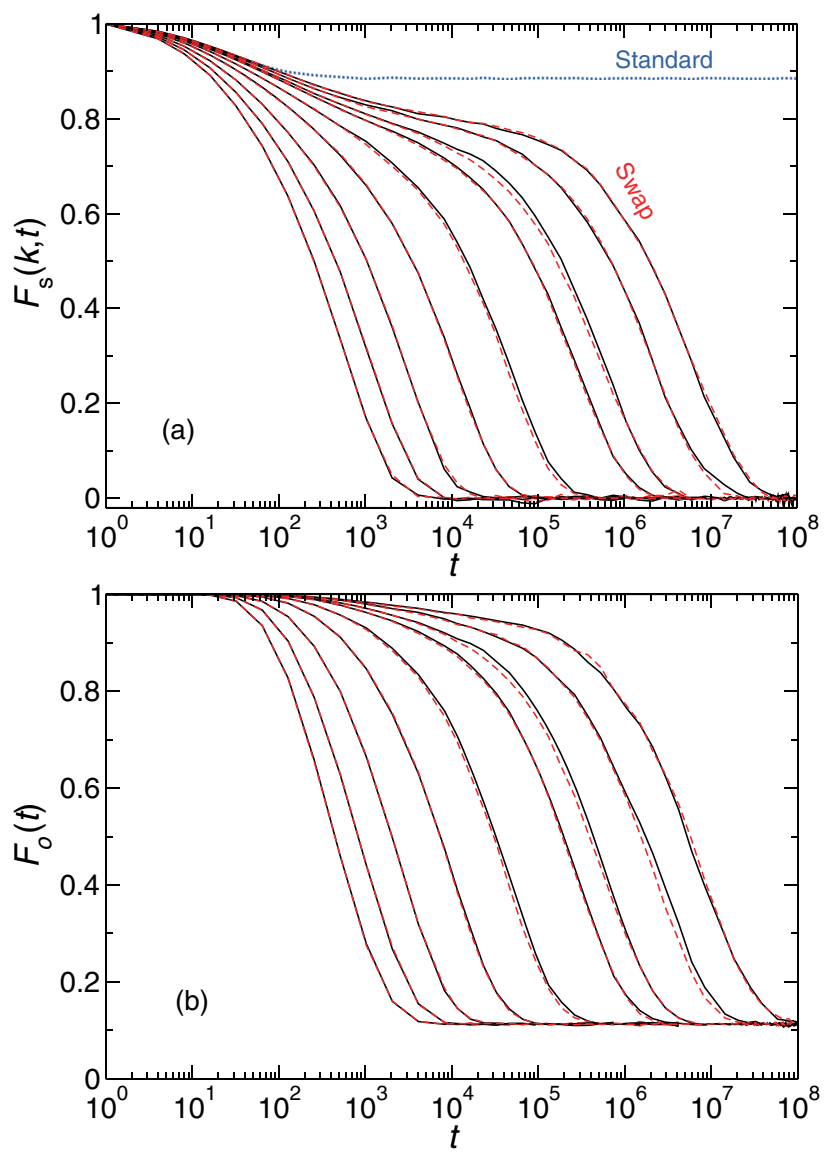

FIG. 4. Swap dynamics in a soft repulsive system of nonadditive particles studied in Sec. IV C with $\epsilon=0.2$. (a) Selfincoherent scattering $F_{s}(k, t)$ computed, respectively, on the first (solid lines) and second (dashed lines) halves of the simulation run. Results for standard dynamics without swap for the lowest temperature are shown with a dotted line. (b) Collective overlap correlation function $F_{o}(t)$. In both panels, temperatures are $T=0.25,0.175,0.125,0.092,0.075,0.065,0.062,0.058$, and 0.0555 , from left to right. Swap Monte Carlo simulations fully decorrelate both single-particle and collective density fluctuations in a regime where standard Monte Carlo simulations may be fully arrested. 
indication that swap dynamics has been correctly implemented and that it provides the correct sampling of phase space. The second information gained from this set of data is the clear indication that the swap Monte Carlo algorithm extends the regime where equilibrium studies are possible by a large amount and is able to produce highly stable glass configurations.

To validate the equilibration protocol discussed at the beginning of this section, we show in Fig. 4(a) the incoherent scattering function for the same system as in Fig. 2 (see also Sec. IV C) evaluated over the first and the second halves of the simulation at various temperatures. As we can see, the two sets of curves agree within statistical uncertainty over a wide range of temperatures, demonstrating the absence of aging. For the lowest temperature at which thermalization with swap moves is achieved, we show the corresponding relaxation function obtained without swap, which quickly decays to a plateau that extends over the last 6 decades of the simulation. This shows that without swap moves, the dynamics is fully arrested at these low temperatures, and no equilibrium simulations can presently be performed in this regime with conventional computational techniques.

In Fig. 4(b), we show the collective overlap function, Eq. (16), which decorrelates to a density-dependent plateau at long times, as expected in ergodic equilibrium simulations. The two plots of Fig. 4 underline the fact that swap Monte Carlo simulations fully decorrelate both singleparticle and collective density fluctuations in a regime where standard Monte Carlo simulations may be fully arrested and therefore represent an efficient and reliable method to sample the configuration space.

\section{RESULTS FOR DISCRETE MIXTURES}

\section{A. Binary mixtures}

Simple binary mixtures of repulsive spheres were the first computer models for supercooled liquids simulated using the swap Monte Carlo method [36,37]. Here, we focus on the "historical" 50:50 binary mixture introduced long ago by Bernu et al. [68], which has been frequently used since its introduction. The pair interaction is given by Eqs. (5) and (8) with $\epsilon=0$ and $F\left(r_{i j}\right)=c_{\alpha \beta}$, where $\alpha, \beta=A, B$ are species indices. The size ratio is $\sigma_{A} / \sigma_{B}=1.2$, resulting in a polydispersity $\delta=9.1 \%$. The potential is cut off and shifted at a distance $r_{\text {cut }}=\sqrt{3}$, a specific value that was often used in previous studies $[37,47,67]$. We simulate $N=1024$ particles at the number density $\rho=1$.

As already demonstrated before [67], swap moves help to accelerate sampling in this system, even though their acceptance rates is relatively low, of order $a \sim 10^{-2}$. We confirm this finding in Fig. 5, where we compare the structural relaxation time $\tau_{\alpha}$ measured during standard and swap Monte Carlo simulations. Over the range of temperatures at which the system can be equilibrated according to our criteria (see Sec. II D), swap moves result in a speedup

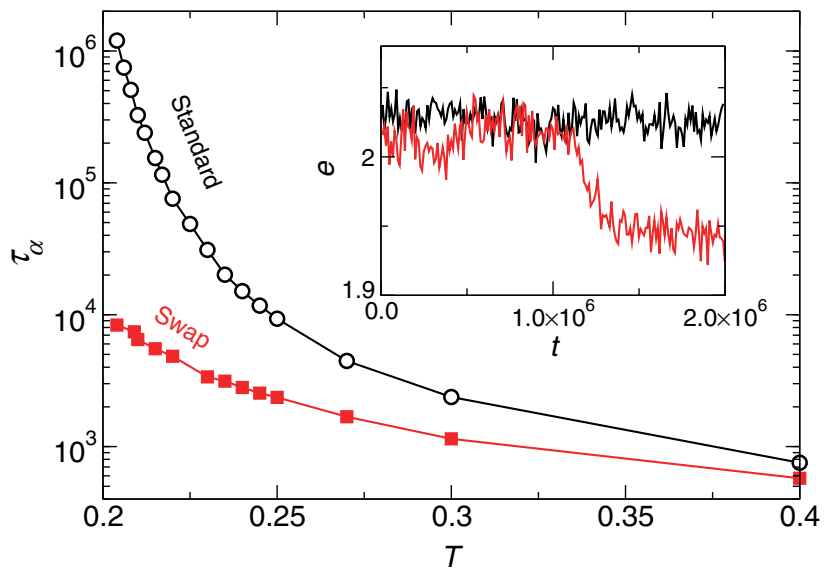

FIG. 5. Relaxation times $\tau_{\alpha}$ of standard (black empty points) and swap (red solid squares) simulations for the binary mixture of soft repulsive spheres studied in Sec. III A. The speedup offered by the swap moves is obvious, but the system is unstable below $T=0.202$ where it crystallizes, and temperatures below the mode-coupling temperature $T_{\mathrm{MCT}}=0.199$ cannot be studied. The inset shows a time series of the potential energy for standard (black) and swap (red) simulations at $T=0.2$, and we see that crystallization is easily observed when swap moves are introduced.

of about 2 orders of magnitude at the lowest temperature. Notice that contrary to published analysis [46], we find that the efficiency of the swap over the standard Monte Carlo method is strongly temperature dependent, and efficiency increases rapidly as temperature decreases.

Unfortunately, however, the temperature range that can be analyzed with this system does not change dramatically when swap moves are introduced. In fact, even using standard MC, the system crystallizes at the lowest studied temperature and becomes unstable when $T<0.202$, which is marginally larger than the location of the mode-coupling crossover, $T_{\mathrm{MCT}} \approx 0.199$. Notice that earlier, incorrect determinations of the mode-coupling crossover temperature of this system have misleadingly suggested that temperatures well below $T_{\mathrm{MCT}}$ could be simulated with this system. In reality, $T_{\mathrm{MCT}}$ represents the lowest temperature that can be safely studied, swap moves merely providing a more efficient way of producing thermalized configuration in the temperature regime $T>T_{\mathrm{MCT}}$. In other words, swap MC accelerates the dynamics of the system but does not allow the exploration of a temperature regime that is not already accessible with ordinary simulations.

In the inset of Fig. 5, we show the time series of the potential energy of a sample at $T=0.2$, where rapid crystallization is observed when swap dynamics is employed. We note that crystallization in this model is well documented and has been studied in detail in small samples $[47,69]$. Since complex strategies would be needed to detect and filter out crystallized configurations [67] already near the mode-coupling crossover, we conclude that this "historical" model can indeed be efficiently simulated using the 
swap Monte Carlo method but is too poor a glass former to fruitfully explore novel physical regimes.

Within the realm of simple binary mixtures, it is difficult to make further progress using swap Monte Carlo simulations because, to increase the structural stability of the system, one would need to increase the size ratio (for instance, using the more stable $\sigma_{A} / \sigma_{B}=1.4$ well-studied model), but this would imply that the already-very-low acceptance rate for swap moves would become vanishingly small and swap would thus not be a useful method. Therefore, the trade-off between stability and swap efficiency leaves very little room for a drastic improvement of simulation techniques when binary mixture models of glass formers are used. Another option is to introduce nonadditivity in the interactions, as, for instance, in the classic KobAndersen mixture [58]. Rather than for binary mixtures, we explore this possibility for a different family of models based on continuously polydisperse particles (see Sec. IV).

\section{B. Ternary mixture}

Given the limits demonstrated above for binary mixtures, a natural strategy is to increase the number of components in the model. Adding more chemical components is indeed a commonly used method to improve the glass-forming ability of metallic alloys. In addition, by increasing the number of components, one can simultaneously increase the polydispersity, and thus the glass-forming ability of the model, while preserving the swap efficiency by introducing particles with size ratios that are small enough for swap moves to be frequently accepted.

This strategy was recently followed in Ref. [50], where a ternary mixture of soft spheres was introduced and studied using swap Monte Carlo dynamics. The potential used in that work can be cast in the form of Eq. (5) with a softness parameter $n=12$ and $F\left(r_{i j}\right)$ as in Eq. (6), with a cutoff distance $r_{\text {cut }}=1.25 \sigma_{i j}$. We simulated systems with $N=$ 1500 particles at the number density $\rho=1.1$, as in the original version of the model [50]. The size ratio between two species is $\sigma_{A} / \sigma_{B}=\sigma_{B} / \sigma_{C}=1.25$, which is slightly larger than for the binary mixture studied above in Sec. III $\mathrm{A}$, and compositions $x_{A}=0.55, x_{B}=0.30$, and $x_{c}=0.15$, which ensures that all species roughly occupy the same fraction of the total volume. The corresponding size polydispersity is $\delta \approx 17 \%$, so we can expect a smaller tendency for the system to crystallize. Simultaneously, we also expect the acceptance of the swap moves to be much smaller than for the binary mixture. In agreement with Ref. [50], we find that the acceptance rate for swaps is of the order $a \sim 10^{-5}$ at low temperatures. To speed up the simulations, we therefore only attempt swap moves between species $(A, B)$ and $(B, C)$ separately because the probability of accepting swaps between pairs of $(A, C)$ particles is negligible.

Despite the low acceptance rate, it was claimed in Ref. [50] that swap moves allow for a dramatic speedup of the thermalization in this model. In the reduced units described above, we locate the mode-coupling crossover temperature near $T_{\mathrm{MCT}} \approx 0.288$, and Gutierrez et al. claim to have achieved thermalization down to $T=0.22 \approx 0.76 T_{\mathrm{MCT}}$. Based on dynamic scaling arguments, they estimate that the relaxation time at $T=0.22$ is $\tau_{\alpha} / \tau_{0} \approx 10^{15}$, where $\tau_{0}$ is the value of the relaxation time near the onset temperature $T_{0}$. Thus, the claim is that swap Monte Carlo provides an increase in the accessible window of relaxation times of about 10 orders of magnitude as compared to standard molecular-dynamics simulations [50].

We have repeated and extended these simulations using standard and swap Monte Carlo dynamics. In Fig. 6, we present the temperature evolution of the structural relaxation times for both these dynamics. We confirm that despite the very low acceptance rate of the swap moves, the speedup of the dynamics produced by these rare swaps is important. For instance, at the lowest temperature simulated by standard Monte Carlo, $T=0.29$, the relaxation time is reduced by a factor of about $10^{2}$ when swap moves are introduced. Following the evolution of $\tau_{\alpha}$ using swap moves, we find, however, that $\tau_{\alpha}$ becomes too large to be accurately measured for $T \leq 0.24$, and particles in fact barely move over the entire simulation performed at $T=0.22$. Therefore, we conclude that our swap Monte Carlo simulations fail to thermalize the model for $T \leq 0.24$. In Ref. [50], thermalization was tested by reweighting the probability distribution functions of the potential energy. We could reproduce this thermalization test in our work, thus demonstrating that this test fails to

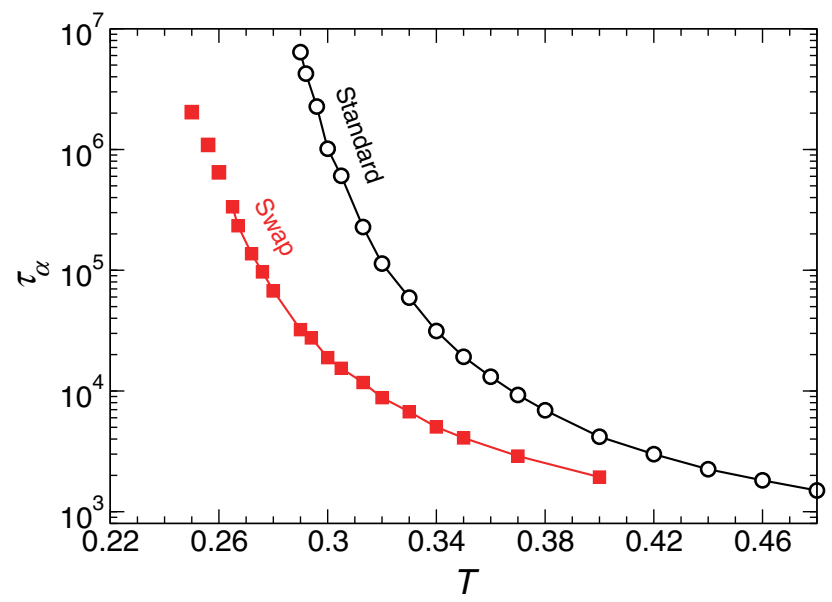

FIG. 6. Relaxation times $\tau_{\alpha}$ of standard (black empty points) and swap (red solid squares) simulations for the ternary mixture of soft repulsive spheres studied in Sec. III B. The speedup offered by the swap moves is obvious, but the system is unstable below $T=0.26 \approx 0.9 T_{\mathrm{MCT}}$ where it demixes and crystallizes. Disconnected squares are a rough estimate of $\tau_{\alpha}$ obtained using short simulations in the unstable region. Stable and equilibrated states can be accessed down to $T \approx 0.26<T_{\mathrm{MCT}}=0.288$, extending the dynamic range by about 2 orders of magnitude as compared to standard simulations. 
detect the lack of thermalization and proper sampling for the lowest studied temperatures. Measuring the structural relaxation time and the relaxation dynamics is thus a more accurate and more discriminating thermalization test than techniques based on global static observables only.

In addition to the lack of thermalization at low temperatures, we also find signatures of structural instability of the fluid at even higher temperatures, $T \leq 0.26$. Below this temperature, our criteria for the absence of crystallization or demixing are no longer fulfilled, and the system is eventually unstable within the window of $200 \tau_{\alpha}$ that we use to assess stability. Using shorter time windows before the system crystallizes, we obtain a rough estimate of the relaxation time $\tau_{\alpha}$ in the unstable regime, and we show these results as disconnected squares in Fig. 6. In Ref. [50], the glass-forming ability of the model was not discussed, but there may be evidence of ordering in the reported peak of the specific heat. An alternative reason for the absence of ordering in the data of Ref. [50] is that the performed simulations covered a smaller time window of about $10^{6}-10^{7}$ Monte Carlo sweeps, whereas we simulate up to $10^{9}$ sweeps in our work. Of course, preventing ordering through shorter simulations implies that thermalization becomes more difficult to achieve, and an accurate sampling of phase space is then problematic.

Comparing the stable results for the ternary mixture to the ones of the binary mixture in Fig. 5, it is clear that the efficiency of swap Monte Carlo is essentially preserved and that thermalization and metastability of the fluid branch have indeed been extended to temperatures below $T_{\mathrm{MCT}}$, although the gain is far less spectacular than the one claimed in Ref. [50], once thermalization and structural stability are more precisely characterized.

We studied more carefully the structural properties of the ternary mixture using partial structure factors, and we show some representative results in Fig. 7 for $S_{C C}(k)$ at various temperatures. At high temperatures, $T>0.30$, we find that the structure factor resembles the one found for ordinary glass-forming models, with a strong first diffraction peak corresponding to the interparticle distance and a featureless plateau at lower wave vectors. In the low-temperature regime, where swap Monte Carlo is mandatory to achieve thermalization, $0.26<T \leq 0.29 \approx T_{\mathrm{MCT}}$, we find that $S_{C C}(k)$ increases more strongly as $k$ decreases towards 0 , which suggests that composition fluctuations are already quite strong in this regime. Even for these state points, which we deemed "stable" based on our stability criterion, longer simulations show that these fluctuations can trigger a demixing in the system, as illustrated for $T=0.267$ in Fig. 7. We have obtained similarly demixed configurations for temperatures up to $T=0.27$, showing that stability is a real issue in this model. Finally, for $T \leq 0.26$, the system always demixes within our simulation time, which produces a strong low- $k$ peak in the structure factor (see Fig. 7). When the particles are segregated, they then easily

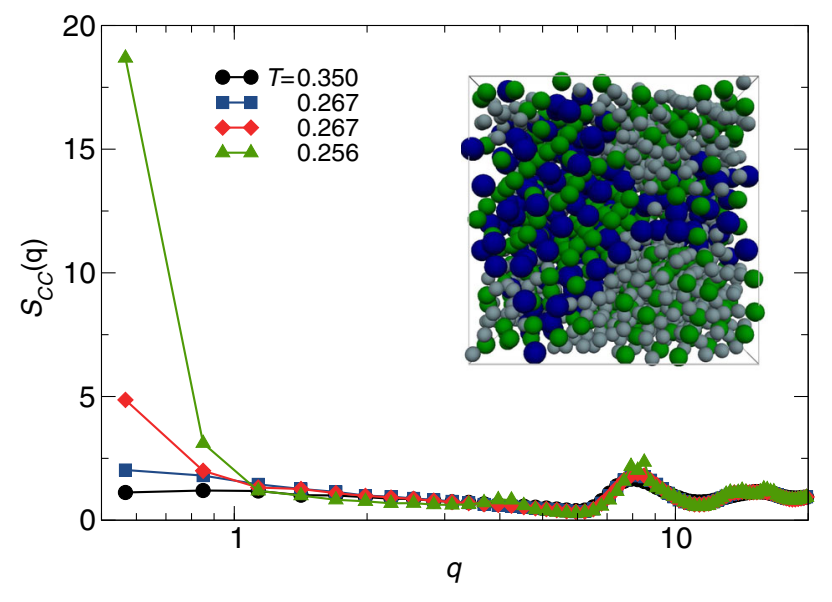

FIG. 7. Partial structure factor $S_{C C}(q)$ for small particles in the ternary mixture of Sec. III B. It is featureless at high enough temperatures, $T=0.350$, and displays strong composition fluctuations at low $k$ in the fluid at $T=0.267$, which may eventually lead to a demixed state at long times. For $T \leq 0.26$, the system is demixed, as shown for $T=0.256$. The inset shows a representative snapshot of a demixed and partially crystallized system at $T=0.256$.

crystallize, and we obtain configurations such as the ones shown in the inset of Fig. 7. We conclude that maintaining this system in a metastable fluid state at low temperatures, $T \leq 0.26 \approx 0.9 T_{\mathrm{MCT}}$, is actually very difficult because (i) thermalization becomes prohibitively difficult and (ii) simulations longer than the thermalization time in this regime produce demixed and ordered configurations.

Finally, to more quantitatively assess the gain in efficiency obtained for this ternary mixture, we have fitted our dynamic relaxation data from standard Monte Carlo simulations to various fitting formula commonly used in the glass literature. Using both a Vogel-Fulcher fit, Eq. (13), which presumably overestimates the data at low $T$, and a parabolic singularity-free formula, Eq. (14), we consistently obtain that the relaxation time at $T=0.26$ is about $\tau_{\alpha} \approx 10^{9}$. This result is 2 orders of magnitude slower than the lowest temperature simulated without the swap moves (see Fig. 6). At this low temperature, $T=0.26$, the relaxation time using swap moves is $\tau_{\alpha} \approx 5 \times 10^{5}$, so the thermalization speedup due to swap moves is about 3 orders of magnitude.

Therefore, we confirm that the ternary mixture introduced in Ref. [50] can be equilibrated at temperatures below the mode-coupling crossover, and we have estimated that this corresponds to an extension of the accessible dynamic regime of about 2 decades compared to standard simulations. This achievement thus competes favorably with the other computational approaches described in the Introduction, but it still does not allow for an exploration of glass physics much closer to the experimental glass transition, which we achieve below for continuous polydispersity. 


\section{Five-component mixtures}

Because ternary mixtures offered only limited success, we tried to improve both swap move acceptance and the metastability of the liquid phase by performing an exploratory study using two different five-component mixtures. We again adjusted the concentration of the various species so that each component roughly occupies the same volume, and we chose the size ratio between the different families to be small enough that swap moves are accepted with a reasonable acceptance rate.

We studied two systems with diameters roughly linearly spaced between $\sigma_{\min }=0.847$ and $\sigma_{\max }=1.333$ for a polydispersity $\delta=16 \%$, and between $\sigma_{\min }=0.826$ and $\sigma_{\max }=1.771$ for a polydispersity $\delta=23 \%$. Thanks to the reduced size ratio between individual components, the swap acceptance rate increased considerably as compared to the binary and ternary mixtures studied above, and it ranged between $a \approx 10 \%$ and $a \approx 20 \%$ depending on temperature. However, both models displayed a strong tendency to demix during swap Monte Carlo simulations, and it proved impossible to equilibrate these systems well below $T_{\mathrm{MCT}}$ following the criteria described above.

We have clearly not exhausted all possible discrete models of glass formers, as the parameter space becomes very large when the number of components increases. It is possible that some parameter combination provides both a rapid thermalization and a good glass-forming ability, and more work would be needed to explore this hypothesis in a more systematic manner, as done, for instance, in the context of simplified models of bulk metallic glasses [70].

\section{CONTINUOUSLY POLYDISPERSE SYSTEMS}

\section{A. Why continuous polydispersity?}

The difficulties highlighted in the previous sections arise from the interplay of several competing effects. Reducing the diameter difference between species improves the acceptance of swap moves and thus accelerates thermalization, but the resulting reduced polydispersity makes the system prone to crystallization. Additionally, we found that simple multicomponent mixtures show an important tendency to demixing at low temperatures.

To tackle these issues at once, we considered a class of models characterized by a continuous particle size distribution $P(\sigma)$. In such systems, swap moves are more likely to be accepted because there always exist pairs of particles whose diameters are sufficiently close to one another. We found that the succession of a large number of successful swaps between pairs with similar diameters actually facilitates the thermalization of the system. Physically, the end result is that the diameter of each particle performs a kind of random walk in diameter space. An efficient exploration of the diameter distribution seems to be the key for efficient thermalization, as discussed further below in Sec. V.
In addition, by choosing a sufficiently high degree of polydispersity, it may be possible to stabilize the liquid against crystallization and fractionation. Therefore, wellchosen continuous particle size distributions seem able to solve all problems encountered in Sec. III for discrete mixtures at once.

In this section, we study models in which particles interact via Eq. (5), with the cutoff function $F\left(r_{i j}\right)$ given by Eq. (6) and a cutoff distance $r_{\text {cut }}=1.25 \sigma_{i j}$. We simulate $N=1500$ particles at $\rho=1$. We also fix the particle size distribution to be of the form given by Eq. (2) and vary parameters such as the pair potential and its additivity. This particle size distribution is controlled by a unique parameter, the size ratio $\sigma_{\max } / \sigma_{\min }$ or, equivalently, the size polydispersity $\delta$. Using insight from preliminary studies on hard sphere systems [51], we fix $\sigma_{\max } / \sigma_{\min }=2.219$, which implies $\delta \approx 23 \%$. This observation is in qualitative agreement with the earlier results of Fernandez et al. [43], who simulated repulsive spheres with a flat size dispersion and found an optimal stability for polydispersities in the interval 20\%-30\%. In these models, the acceptance of swap moves is typically very high, $a \sim 20 \%-30 \%$, and does not change dramatically with temperature.

\section{B. Influence of the particle softness}

Our first analysis of models with continuous polydispersity concerns the role of the particle softness. Previous studies have found that softness can have a nontrivial impact on glass properties, such as fragility [71] and glassforming ability [57]. Here, we simulated polydisperse soft particles, varying the softness exponent $n$ using the values $n=8,12,18$, and 24 . We focus on a continuously polydisperse model with additive interactions, $\epsilon=0$. In the limit where $n \rightarrow \infty$, the model becomes equivalent to the hard sphere model studied in Ref. [51], which displays excellent stability and efficient thermalization. Therefore, the present family of models appears as the natural extension of these hard sphere results to soft potentials.

In Fig. 8, we show the structural relaxation times as a function of the temperature for different softness exponents obtained from both standard and swap Monte Carlo simulations. Temperatures are scaled by the corresponding mode-coupling temperature $T_{\mathrm{MCT}}$ of each model, so different systems can be represented in the same graph. In all these systems, swap moves speed up thermalization significantly. As the exponent $n$ increases, i.e., as repulsive forces get steeper, it becomes possible to equilibrate the system at increasingly lower temperatures relative to $T_{\mathrm{MCT}}$.

In addition, as for discrete mixtures, the models presented in this section also have a tendency to demix at low temperatures. We have again carefully checked the low- $k$ behavior of the partial structure factors, which informs us about the presence of large composition fluctuations. Despite the improved stability, we found that the system may still demix at low enough temperatures during the 


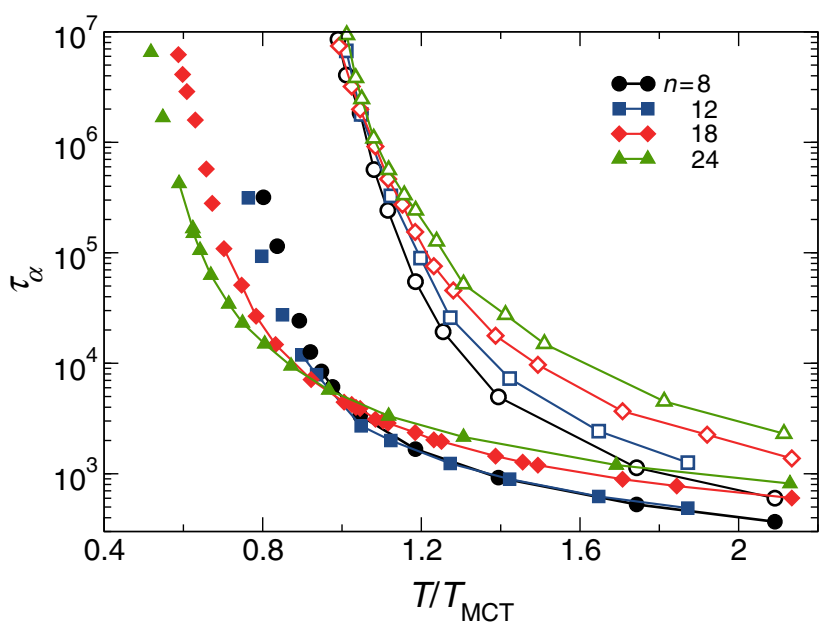

FIG. 8. Relaxation times for continuously polydisperse systems of repulsive soft spheres with different softness exponents $n=8$, 12,18 , and 24 . Temperatures are scaled by $T_{\mathrm{MCT}}$ to allow direct comparison between models, with $T_{\mathrm{MCT}}=0.143,0.267,0.468$, and 0.662 , respectively. Open symbols represent the standard Monte Carlo dynamics, and closed symbols show the swap algorithm, for which unconnected symbols represent structurally unstable state points where only a rough estimate of $\tau_{\alpha}$ is obtained in short simulations. A larger $n$ yields better efficiency and better structural stability.

swap Monte Carlo simulations. For these unstable state points, we again use shorter simulations to obtain a rough estimate of the relaxation time, and we show these data using disconnected symbols in Fig. 8. Regarding the structural stability of the models, there is again a clear trend with the softness exponent $n$. We find that softer systems are more prone to structural instability than harder ones. We emphasize that this result is most likely system specific since the opposite trend was found in other models of glass formers [57,72].

Combining the effect of a more efficient thermalization and a better stability of the fluid state, we conclude that models with larger $n$ values represent the best choice of parameters within the present family, the system with $n=24$ being stable and efficiently thermalized down to $T \approx 0.6 T_{\mathrm{MCT}}$ (see Fig. 8). The trend that we find suggests that a system of polydisperse hard spheres with $n=\infty$, such as the one recently simulated in Ref. [51], might actually prove to be the best glass former in this class of systems, with continuous polydispersity, repulsive interactions, and additive interactions.

\section{Nonadditive interactions}

To suppress the tendency to demixing while preserving a continuous form of polydispersity, we have introduced nonadditivity in the potential by modifying the sum rule for particle diameters, as described in Eq. (8). Nonadditive interactions are known to stabilize the liquid in metallic alloys [57,58]. Moreover, this effect has been demonstrated explicitly in nonadditive hard spheres $[73,74]$. Physically, choosing $\epsilon>0$ frustrates phase separation because particles of different diameters can now stay closer to one another than in the additive case with $\epsilon=0$. To study the effect of nonadditivity, we set the softness exponent to $n=12$, and we vary $\epsilon$ using $\epsilon=0.0,0.1,0.2$, and 0.3 . Note that some results for the case $\epsilon=0.2$ have already been presented in Sec. II, when discussing the details of the swap algorithm and the thermalization checks.

Figure 9 shows the structural relaxation times obtained by varying the degree of nonadditivity. As in the previous section, we scale the temperatures by the measured $T_{\mathrm{MCT}}$ for each model. For comparison, we also redraw the results for the additive system $(\epsilon=0)$ with $n=12$. Regarding thermalization efficiency, we find that nonadditivity improves the performance of the swap algorithm, as lower temperatures relative to $T_{\mathrm{MCT}}$ can be studied when $\epsilon>0$, but it is not easy to provide a detailed physical understanding of this result.

Analysis of the low- $k$ behavior of the partial structure factors $S_{\alpha \alpha}(k)$ shows that phase separation is strongly suppressed by the nonadditivity for both $\epsilon=0.1$ and 0.2 . At low temperatures, however, the system can crystallize, as detected from drops in the time series of the energy and from the appearance of well-defined peaks in the structure factor, and we could frequently observe these crystallization events for $\epsilon=0.2$ and 0.3 . For $\epsilon=0.1$, the liquid remains stable down to the lowest temperature that we could equilibrate with the swap Monte Carlo. This is in

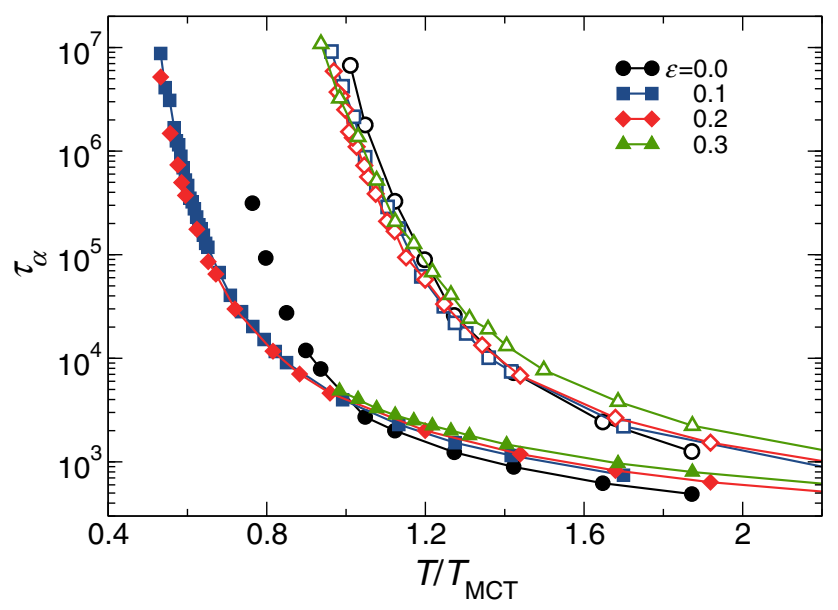

FIG. 9. Relaxation times for systems with continuous polydispersity, $n=12$, and different nonadditivity parameters $\epsilon$. Temperatures are scaled by $T_{\mathrm{MCT}}$ to allow direct comparison between models, with $T_{\mathrm{MCT}}=0.267,0.176,0.104$, and 0.0534, respectively. Open symbols represent the standard Monte Carlo dynamics, and closed symbols show the swap algorithm, for which unconnected symbols represent structurally unstable state points where only a rough estimate of $\tau_{\alpha}$ is obtained in short simulations. A well-chosen amount of nonadditivity, $\epsilon \approx 0.1-0.2$, considerably improves the efficiency of thermalization and the structural stability. 
fact the only model among the eleven models studied in this paper that perfectly fulfills our equilibration and stability criteria throughout the entire accessible temperature range.

The existence of an optimal nonadditivity parameter $\epsilon$ for glass-forming ability presumably results from a competition between demixing, which takes place when $\epsilon=0$, and crystallization, for large $\epsilon$. We suggest that these two distinct paths to ordering actually compete for $\epsilon=0.1-0.2$, which results in an enhanced frustration and thus a higher structural stability. Similar physical arguments have been proposed in Ref. [70] to explain the glass-forming ability of simple nonadditive mixtures.

Whereas the additive model with $n=12$ was not the best choice of softness in the previous section, we find that including nonadditivity significantly improves both the efficiency of the swap algorithm and the structural stability of this model. We suggest that models with larger $n$ (including hard spheres) with nonadditive diameters would be even better choices.

It is interesting to contrast the results for the $n=12 \mathrm{soft}$ repulsion using a continuous size distribution and a nonadditivity $\epsilon=0.1$ to the results obtained for the binary mixture in Sec. III A for the same pair potential. The conclusion is that the thermalization and stability limits have been decreased from $T \approx T_{\mathrm{MCT}}$ for the binary mixture down to $T \approx 0.5 T_{\mathrm{MCT}}$ for the present system. This represents a major methodological improvement that we try to quantify in terms of time scales in the next subsection.

\section{Experimental time scales are matched by simulations}

In the previous section, we showed that by optimizing the additivity and the form of the pair potential, temperatures as low as $T \approx 0.5 T_{\mathrm{MCT}}$ could be thermalized using swap Monte Carlo in the metastable fluid state. Because ordinary simulations stop near $T \approx T_{\mathrm{MCT}}$, one may wonder how large the corresponding gain is in terms of structural relaxation times. It is relatively easy to answer this question when the improvement is modest, but this becomes a delicate task in our case, as thermalization is achieved in a temperature regime where the standard dynamics is completely frozen in our observation window and where equilibrium time scales can only be obtained by extrapolation. Extrapolating time scales down to the lowest temperatures where the swap Monte Carlo method can thermalize may depend sensitively on the fitting procedure, and it therefore requires some care.

We have devised a robust strategy that answers the following question for each model: Is the thermalization speedup due to the swap Monte Carlo algorithm large enough to fill the eight-decade gap between ordinary simulations and experiments? To answer this question for a given model, we employ ordinary Monte Carlo simulations to access a range of relaxation times up to $\tau_{\alpha} / \tau_{0} \approx 10^{4}$, where $\tau_{0}$ represents the value of $\tau_{\alpha}$ at the onset of glassy dynamics. In experiments [5], the glass temperature $T_{g}$ corresponds to the value $\tau_{\alpha} / \tau_{0} \approx 10^{12}$, which we take as our practical definition of $T_{g}$. Using the various functional forms described in Sec. II B, we realized that estimating the location of $T_{g}$ from numerical measurements of $\tau_{\alpha}$ is actually possible with modest uncertainty. More precisely, for a given model, we use all three functional forms in Eqs. (13)-(15) to estimate the location of $T_{g}$ from the definition $\tau_{\alpha}\left(T_{g}\right) / \tau_{0}=10^{12}$. Despite the qualitative differences between these functional forms, the range of $T_{g}$ values is reasonably small, typically $\Delta T_{g} / T_{g}=\left(T_{g}^{\mathrm{VFT}}-T_{g}^{\text {Arrhenius }}\right) /\left(2 T_{g}^{\text {parabolic }}\right) \approx 12 \%$, with minor variations from one model to the other. Because the VFT law tends to overestimate the increase $\tau_{\alpha}$ and the Arrhenius law underestimates it, these two forms respectively provide an upper and a lower bound to the real location of $T_{g}$, while estimates from the parabolic law usually fall between those two bounds. Elmatad et al. [63] have shown that the parabolic law accounts for the variation of relaxation times of glass-forming liquids over a broad range of temperatures, ranging from the onset down to the laboratory glass transition. We therefore expect our parabolic extrapolation to provide a reasonable determination of $T_{g}$, and the other two temperatures to provide a solid estimate of the interval of confidence.

We illustrate this procedure in Fig. 10(a) where we show the temperature evolution of $\tau_{\alpha}(T)$ from standard Monte Carlo dynamics for the nonadditive model studied in Sec. IV C, using an Arrhenius representation. We fit these Monte Carlo dynamic data and estimate three different locations for $T_{g}$, which delimit the range of possible values for the location of $T_{g}$, highlighted with the vertical dashed lines. We then show the evolution of the relaxation time for metastable fluid states when swap Monte Carlo moves are used in the same Arrhenius representation. We find that the swap relaxation time remains modest in the vicinity of $T_{g}, \tau_{\alpha} / \tau_{0} \approx 10^{2}-10^{3}$ and that this particular system can be thermalized and kept metastable at temperatures even below the experimental glass temperature $T_{g}$. This finding has several important consequences.

(i) The speedup of the thermalization at $T_{g}$ is of about $10( \pm 1)$ orders of magnitude, implying that computer simulations can now comfortably study that temperature regime in thermal equilibrium.

(ii) The maximal speedup obtained with the swap Monte Carlo is in fact much larger than these 10 orders of magnitude because temperatures lower than $T_{g}$ can be thermalized, but estimating that gain becomes very sensitive to the chosen extrapolation.

(iii) For selected models, we can now access a temperature regime that even experiments cannot reach, thus opening a novel observational window on the physics of glasses in a regime that has never been probed before, either experimentally or numerically. 

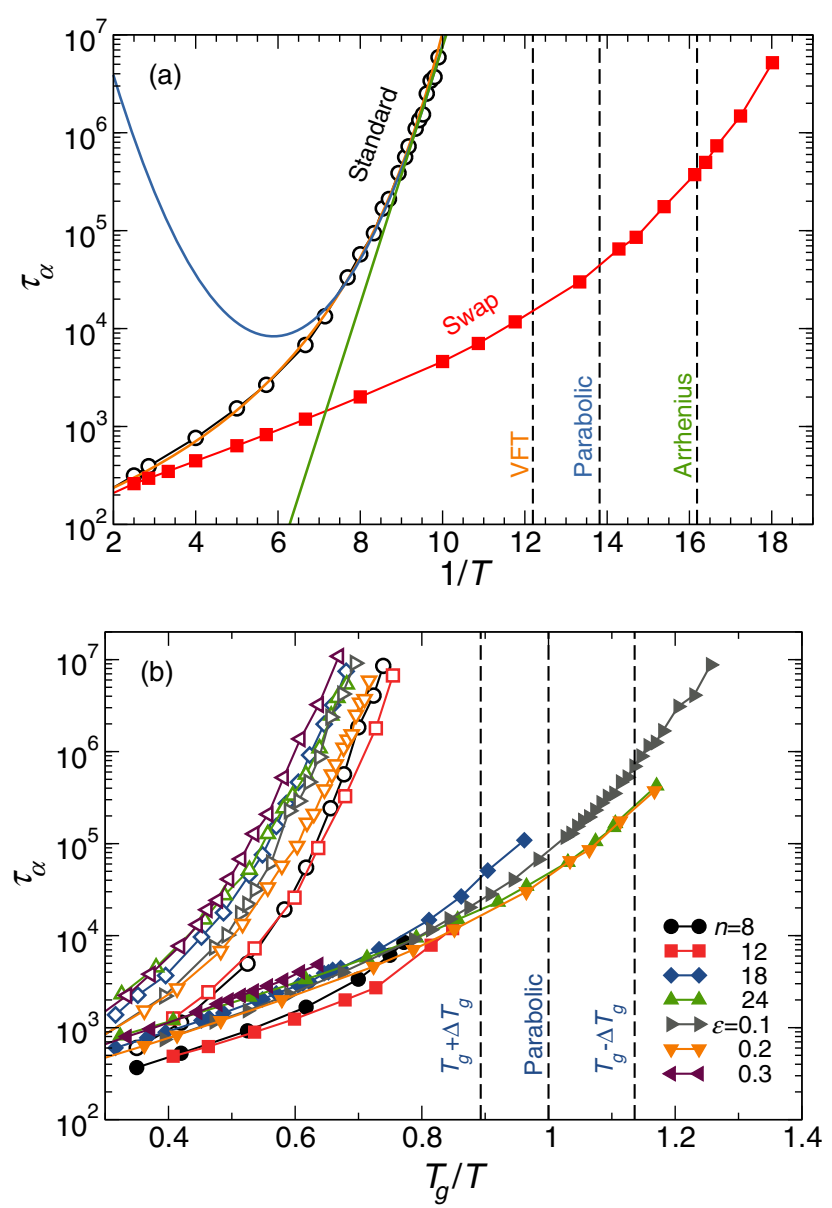

FIG. 10. (a) Relaxation times for the nonadditive model with $n=12$ and $\epsilon=0.2$ for standard and swap Monte Carlo dynamics. The standard dynamics is fitted with the VFT, parabolic, and Arrhenius laws, as shown with lines, which are used to estimate the location of the experimental glass temperature $T_{g}$, as shown with vertical dashed lines. For this system, the swap dynamics is able to provide stable and thermalized configurations at temperatures below $T_{g}$. (b) Relaxation times obtained from standard (open symbols) and swap (filled symbols) dynamics for various size polydisperse models of various softness $(n)$ and nonadditivity $(\epsilon)$ are shown in an Arrhenius form with rescaled temperature $T_{g} / T$, where $T_{g}$ is estimated as in (a). For all models, the thermalization speedup near $T_{g}$ is of about 10 orders of magnitude, some models being structurally stable down to temperatures below $T_{g}$.

To quantify the performance of the swap algorithm, we estimate the range of $T_{g}$ values for each model studied in this section and use these fitted values to construct an Angell plot, representing the logarithm of the relaxation times as a function of the scaled inverse temperature, $T_{g} / T$ [see Fig. 10(b)]. In practice, we use the value given by the parabolic fit, which falls in the middle of the fitted range, and show the corresponding uncertainty, estimated from VFT and Arrhenius fits, with the vertical dashed lines in Fig. 10(b). Standard Monte Carlo simulations typically stop near $T / T_{g} \approx 1.3-1.5$, in the vicinity of the mode-coupling crossover. For most models, the swap algorithm performs so well that the thermalization time at $T_{g}$ remains modest, $\tau_{\alpha} / \tau_{0} \approx 10^{2}$. This corresponds to a thermalization speedup at $T_{g}$ of 10 orders of magnitude. As mentioned before, models with soft potentials and additive interactions are prone to structural instability, and some of them are not stable down to $T=T_{g}$. However, for several models, we find that thermalization and fluid metastability can be maintained below $T_{g}$.

The discovery of such glass-forming models associated with an efficient algorithm to thermalize them represents the main achievement of our work.

\section{MICROSCOPIC INSIGHTS INTO THE SWAP DYNAMICS}

\section{A. Dynamics of particle diameters}

The previous sections demonstrated that swap Monte Carlo moves can enhance thermalization by several orders of magnitude, the effect being most spectacular in continuously polydisperse systems, for which swap moves have a very high acceptance rate. In this section, we shed some light on the microscopic mechanisms that are responsible for this acceleration. We carry out this analysis for a nonadditive polydisperse model with $\epsilon=0.2$ introduced in Sec. IV C.

Previously, it has been suggested that the swap moves increase the particle mobility because they allow the particles to escape the cage formed by their neighbors $[37,50]$ after a nonlocal swap move. This view seems correct when one considers that particles exchange their positions because a caged particle indeed appears to jump instantaneously to a novel position. However, the swapped particle is actually replaced by another particle which then occupies the caged position itself, and it jumps to a position where another particle is caged, too. Therefore, it is not clear that the cage is affected at all after a swap move, and this simple explanation cannot explain the speedup of the dynamics.

This conclusion is more easily grasped when one considers, as we do, that particles simply exchange their diameters during a swap move, without changing position. In that case, the diameter of each caged particle slowly fluctuates in time. For continuous polydisperse systems, these time fluctuations take the form of a random walk in diameter space. Therefore, we conclude that it is rather the slow wandering of the diameter of each particle that allows the system to relax more efficiently towards equilibrium. A naive physical explanation would be that a caged particle with a large diameter could start diffusing by shrinking its radius, thus being able to squeeze and escape through a small channel. We now demonstrate that the physics is actually more complicated and more collective than this naive image. 
To see this, let us start with some qualitative observations on the time evolution of the diameter of a tagged particle. We show two typical time series of $\sigma_{i}(t)$ for two different particles in Fig. 11. We rescale the time axis by $\tau_{\alpha}$ to better appreciate how much the particle diameter changes over a relaxation time of the system. On short time scales, the diameter fluctuates around its initial value, while at longer times, it changes and eventually visits all values allowed by the particle size distribution. At low temperatures, these relaxation events occur suddenly and appear as jumps. Overall, this behavior strongly resembles the typical features of glassy dynamics known from real-space analysis of single-particle displacements, very much mimicking the cage effect and hopping motion. To reinforce this analogy, we show the time series of the particle displacements for the same two tagged particles in Fig. 11. As expected, they display periods of immobility separated by rapid jumps.

The comparison of the two panels reveals that some of the sudden jumps in diameter space occur at similar times as the sudden jumps of the particle in real space, which indicates that diameter dynamics can trigger diffusion. However, we can also detect jumps occurring in real space without clear counterparts in diameter space, and vice versa. These observations suggest that changing the diameter of a single particle is not necessarily enough to trigger a rearrangement, and they also suggest that changes in the neighborhood of one particle may be enough to trigger a displacement. Overall, the physical picture is that relaxation in these supercooled states is a collective process, and

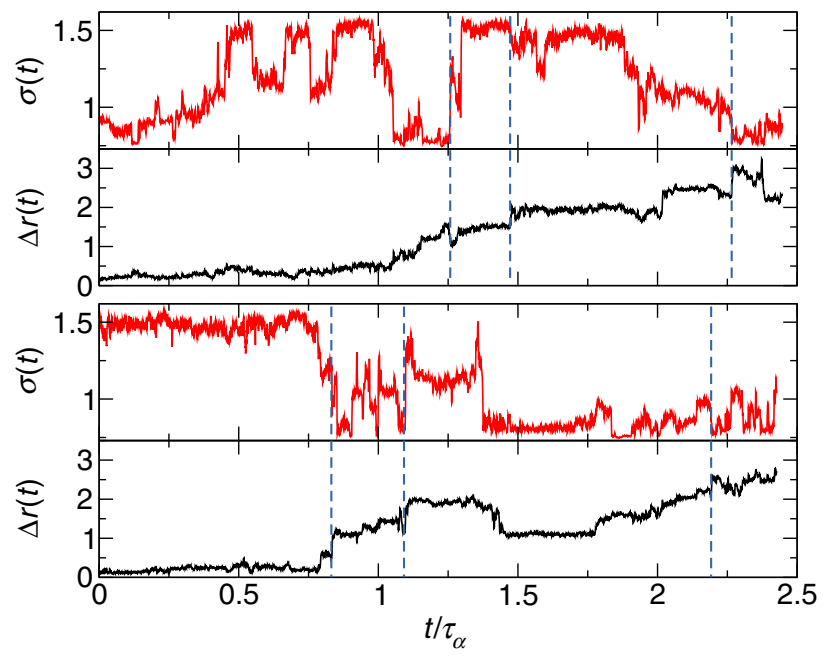

FIG. 11. Time series of individual displacement $\Delta r(t)$ and diameter value $\sigma(t)$ for two tagged particles in the nonadditive model of Sec. IV C with $\epsilon=0.2$ at $T=0.0555$. Intermittent diffusion in real and diameter space is observed, with strong correlations between $\Delta r(t)$ and $\sigma(t)$ highlighted with dashed lines, but we also observe many events in one observable that have no counterpart in the other, indicating that the correlation between the two observables is nonlocal. the efficient thermalization with swap cannot be explained on the basis of a simple single-particle argument.

To quantify the correlation between diameter and position dynamics, we define a time correlation associated with the time evolution of the diameters. We define the following autocorrelation function for diameter fluctuations:

$$
C_{\sigma}(t)=\left\langle c_{\sigma}(t)\right\rangle=\left\langle\frac{\sum_{i} \delta \sigma_{i}(t) \delta \sigma_{i}(0)}{\sum_{i} \delta \sigma_{i}^{2}(0)}\right\rangle,
$$

where $\delta \sigma_{i}(t)=\sigma_{i}(t)-\langle\sigma\rangle$. This function is normalized so that it evolves from unity when $t=0$ to zero at large times when diameters become completely uncorrelated from their initial values. The temperature variation of this function is shown in Fig. 12(a). By lowering the temperature, $C_{\sigma}(t)$ develops a plateau after an initial short time decay and eventually decorrelates at longer times. This confirms the previous qualitative observations that the diameters remain
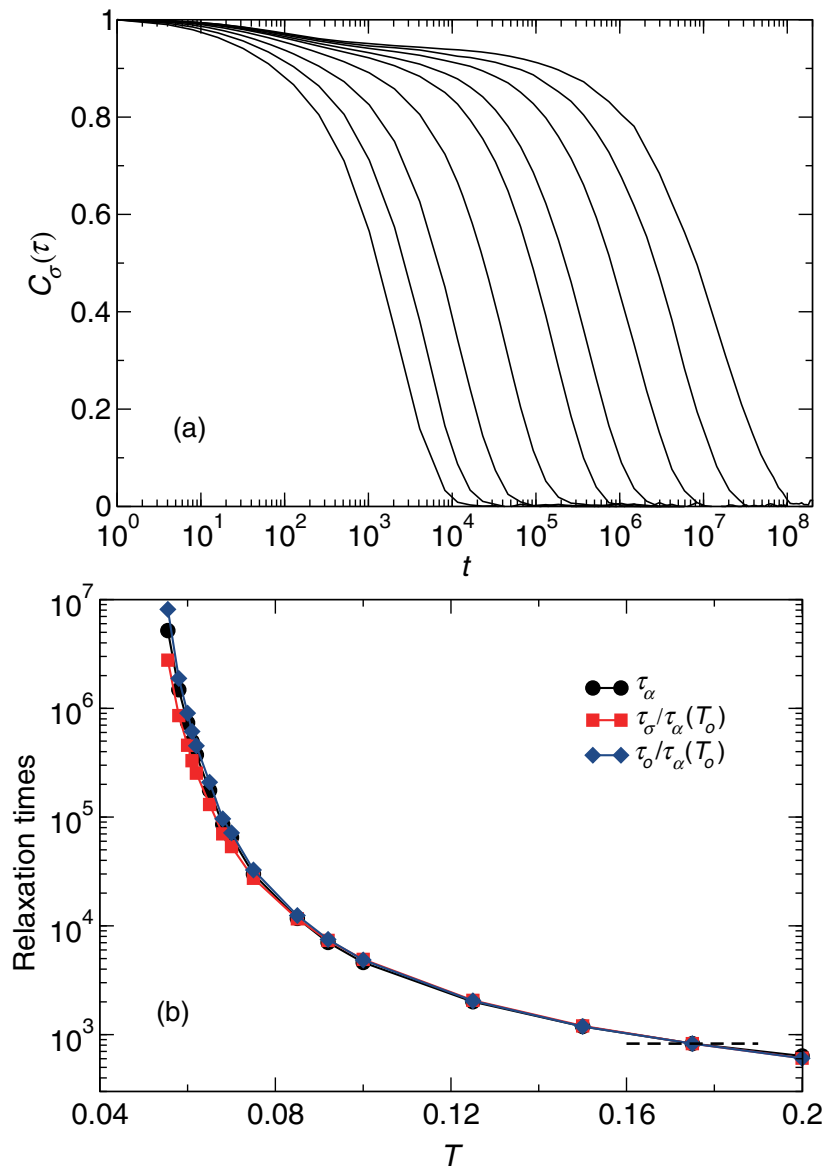

FIG. 12. Nonadditive model of Sec. IV C with $\epsilon=0.2$. (a) Time autocorrelation of particle diameters $C_{\sigma}(t)$ measured during the swap dynamics for temperatures, as in Fig. 4. (b) Relaxation times $\tau_{\alpha}, \tau_{\sigma}$, and $\tau_{o}$ as a function of temperature, with $\tau_{\sigma}$ and $\tau_{o}$ rescaled to coincide with $\tau_{\alpha}$ at $T=0.175$ (shown with the horizontal bar). The three time scales obviously have the same temperature dependence. 
"caged" around some initial value before complete decorrelation.

Let us define the diameter decorrelation times $\tau_{\sigma}$ as the value of time such that $C_{\sigma}\left(\tau_{\sigma}\right)=e^{-1}$. In Fig. 12(b), we compare the temperature evolution of three different relaxation times for single-particle motion $\tau_{\alpha}$, for singleparticle diameter $\tau_{\sigma}$, and for collective density fluctuations $\tau_{o}$. We absorb the observable dependence of these three time scales by rescaling them at a single temperature where the relaxation is fast, namely, $T=0.175$. The striking result of these measurements is that single-particle displacements, density fluctuations, and diameter fluctuations all relax on the same time scale. Because diffusion is fully arrested when the diameters do not fluctuate, we conclude that it is the efficient dynamics in diameter space that drives the structural relaxation in position space and, therefore, the efficient thermalization of the system.

A further intriguing observation about the role of diameter fluctuations stems from the data shown in Fig. 4, where time correlation functions for standard and swap dynamics are compared at the same very low temperature, where even the swap dynamics is very slow. In that case, one observes that the plateau height related to shorttime vibrational motion is different in the two dynamics, the amplitude of these vibrations being much larger when the swap dynamics is used. This observation implies that at short times, the small fluctuations in particle diameters act as an additional degree of freedom that allows each particle to perform back and forth caged motion over a typical distance that is larger than in the standard dynamics. These larger in-cage fluctuations suggest a possible "softening" of local cages, which seems to correlate well with an acceleration of the dynamics. Such a correlation between shorttime motion and structural relaxation is often discussed in the context of glass-forming models [75-78], and it would be interesting to study it further in the present context.

\section{B. Spatially heterogeneous dynamics}

The correspondence between the time scales for diameter and position dynamics, accompanied by a lack of strong correlation at the single particle level, suggests that the physics of diffusion in real and diameter space is cooperative in nature. For instance, diffusive events could happen more easily in a spatial region where the diameter dynamics has been particularly efficient. This hypothesis suggests that we investigate the existence of spatial correlations of the dynamical relaxations.

To illustrate this point qualitatively, we show in Fig. 13 two typical configurations at a temperature $T=0.0555$. We measure the dynamics between an arbitrary initial time $t=0$ and a later time $t=\tau_{\alpha}(T) / 2$. In Fig. 13(a), we show the particles having the $10 \%$ largest displacements in real space over this time lag, whereas in Fig. 13(b), we show the particles having the $10 \%$ largest displacements in diameter space. Particles are drawn using rescaled final diameter
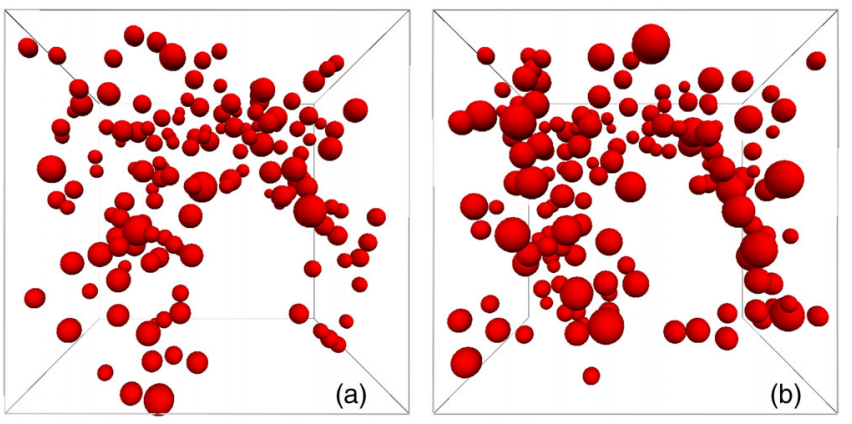

FIG. 13. Snapshots of the $10 \%$ of particles with (a) the largest displacements and (b) the largest diameter change, computed between times $t=0$ and $t=\tau_{\alpha} / 2$ for $T=0.0555$. There is a clear correlation between the spatial regions where dynamics in real and diameter spaces are fast, but the correlation is weak at the single-particle level.

values. We observe a close similarity between regions of faster diffusing particles and regions of particles with large diameter changes, but we also recognize that the correlation does not hold at the particle level. Thus, we conclude that diameter changes and structural relaxation may affect each other in a nonlocal fashion.

The spatial correlations of diameter fluctuations can be characterized using the multipoint functions introduced to study cooperative motion in supercooled liquids [79]. The generic expression of the dynamical susceptibility related to a time-dependent observable $O(t)$ is

$$
\chi_{4}^{O}(t)=N\left[\left\langle O^{2}(t)\right\rangle-\langle O(t)\rangle^{2}\right] .
$$

It quantifies the extent of spatial correlations associated with the local observable $O$ over a time scale $t$ [79]. Here, we measure dynamic susceptibilities associated with both the self-part of density fluctuations, $\chi_{4}^{d}(t)$ with $O(t)=$ $f_{s}(k, t)$ [see Eq. (11)], and with diameter fluctuations, $\chi_{4}^{\sigma}(t)$ with $O(t)=c_{\sigma}(t)$ [see Eq. (17)]. These functions provide information on the spatially heterogeneous dynamics of particle displacements and of diameter changes, and they typically display a peak around the time scales $\tau_{\alpha}$ and $\tau_{\sigma}$, respectively. In Fig. 14, we report the height of these two peaks, $\chi_{4}^{d *}$ and $\chi_{4}^{\sigma *}$, as a function of the temperature. In addition, we also measure and report the behavior of $\chi_{4}^{d *}$ for the standard Monte Carlo dynamics.

This figure provides two main pieces of information. First, we notice that the temperature dependence of $\chi_{4}^{d *}$ in standard and swap simulations is very different. The behavior for standard Monte Carlo is as reported before [80], where $\chi_{4}^{d *}$ increases rapidly from a value $\chi_{4}^{d *} \approx 1$ when the temperature is decreased below the onset $T_{o} \approx 0.18$ to a value $\chi_{4}^{d *} \approx 12$ when approaching $T_{\mathrm{MCT}}$, in a way that mirrors the evolution of the relaxation time $\tau_{\alpha}$, as demonstrated in Fig. 14. The traditional interpretation is that dynamics becomes spatially correlated over larger length 


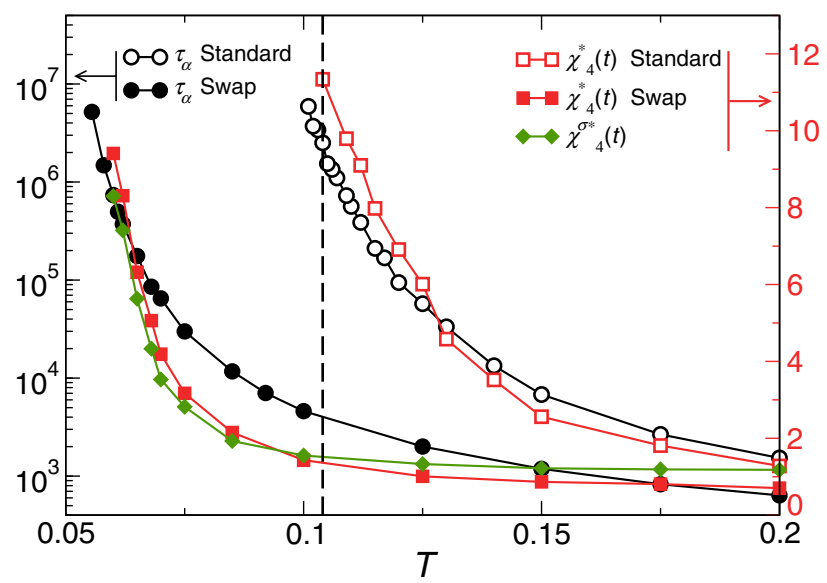

FIG. 14. Temperature evolution of dynamic susceptibilities (right axis) and relaxation times (left axis). The vertical dashed line is $T_{\mathrm{MCT}}=0.104$. Whereas $\chi_{4}^{d}(t)$ grows together with $\tau_{\alpha}$ in standard simulations, $\chi_{4}^{d}(t)$ and $\chi_{4}^{\sigma}(t)$ behave similarly and have a very different temperature dependence which instead mirrors the evolution of the swap relaxation time.

scales as the temperature is lowered. A striking observation is that the swap dynamics near $T_{\mathrm{MCT}}$ displays essentially no spatial dynamic correlations. By construction, swap moves can affect the dynamics of the system but not its equilibrium static properties. Therefore, we conclude that the growth of the spatial correlations detected by $\chi_{4}^{d *}$ for $T>$ $T_{\mathrm{MCT}}$ in standard dynamics is mostly of dynamic, rather than structural, origin. This finding, which agrees qualitatively with previous conclusions $[81,82]$, may also explain why the swap algorithm can be very efficient: If spatial correlations have a strong structural component, then a strong numerical acceleration would likely necessitate the introduction of a more collective algorithm.

The second key point from Fig. 14 is that both quantities $\chi_{4}^{d *}$ and $\chi_{4}^{\sigma *}$ with swap dynamics are quantitatively very close, which confirms that fluctuations in real and in diameter spaces are strongly correlated. Even though the correlation is not strong at the local scale, diameter fluctuations display the same temperature evolution as dynamic heterogeneities. In addition, both quantities follow the growth of the swap relaxation time, the swap algorithm becoming slow at low enough temperatures, at which important spatial correlations of the diameter dynamics are needed to relax to system towards equilibrium.

\section{IDEAS FOR THE FUTURE DESIGN OF GLASS-FORMING MODELS}

In this section, we build on the detailed level of understanding of the swap mechanism reached in the previous sections to propose novel directions and ideas to design new glass-forming models for which the swap Monte Carlo approach could be very efficient.

\section{A. "Hybrid" models for binary mixtures}

A large number of models studied in the past were based on discrete mixtures $[58,68,83]$, as studied in Sec. III. We concluded that the swap method was not well suited for binary mixtures because a large acceptance rate for the swap seems incompatible with a good structural stability. We now show that it is possible to construct models that have the characteristics of binary mixtures and reasonable stability and that can be efficiently simulated using the swap algorithm.

Our idea is to introduce what we call a "hybrid" particle size distribution, as sketched in Fig. 1. These distributions are composed of two main peaks that are a good representation of an $A-B$ binary mixture. In order to have a good glass-forming ability, we choose an equal concentration of particles in these two peaks, and, more importantly, we choose a size ratio that is large enough to avoid the crystallization observed otherwise. Because such a largesize ratio implies that $(A, B)$ swap moves are always rejected, we introduce a third specie in the model, associated with a flat continuous distribution of particle sizes that smoothly connects the two main peaks of the binary mixture. The main idea is that a particle belonging to one of the two main components can be swapped with particles belonging to the intermediate third specie, and it can then slowly tunnel through to reach the other specie. In other words, whereas a direct particle exchange between $A$ and $B$ is unlikely, the addition of the interpolating specie facilitates such exchanges, which can then happen via a large number of intermediate swaps that all have a large acceptance rate.

In practice, we introduce two species $(A, B)$ with flat continuous polydispersity around two average diameter values $\left(\sigma_{A}, \sigma_{B}\right)$ such that $\sigma_{B} / \sigma_{A}=1.6$. We add a third specie, $C$, with an average diameter value $\sigma_{C}=\left(\sigma_{A}+\sigma_{B}\right) / 2$, which continuously interpolates between small and large diameters. Each specie contains roughly $\frac{1}{3}$ of the particles. The final size distribution is described by Eq. (3), with the chosen parameters $x_{A}=0.33, x_{B}=0.34, x_{C}=0.33, \sigma_{A}=0.76$, $\sigma_{B}=1.23, \quad \sigma_{C}=1.00, \quad b_{A}=0.04, \quad b_{B}=0.04, \quad$ and $b_{C}=0.26$. The two-body potential is given by Eqs. (5) and (6), with $n=12$ and a cutoff distance of $r_{\text {cut }}=1.25 \sigma_{i j}$. We perform simulations with $N=1000$ at $\rho=1.3$. With these parameters, the polydispersity is $\delta=20 \%$.

Results for the relaxation times are presented in Fig. 15. As usual, we use disconnected points to represent unstable state points for which short simulations are used to estimate $\tau_{\alpha}$. For this system, again, we observe that equilibration is easily attainable for temperatures below $T_{\mathrm{MCT}}$, and resistance to ordering is ensured down to relatively low temperatures, $T \approx 0.75 T_{\mathrm{MCT}}$. However, at lower temperatures, the system again presents instabilities because of the tendency towards phase separation, which we observe through low- $k$ values of the structure factor. Preliminary results indicate that using nonadditive interactions will most certainly 


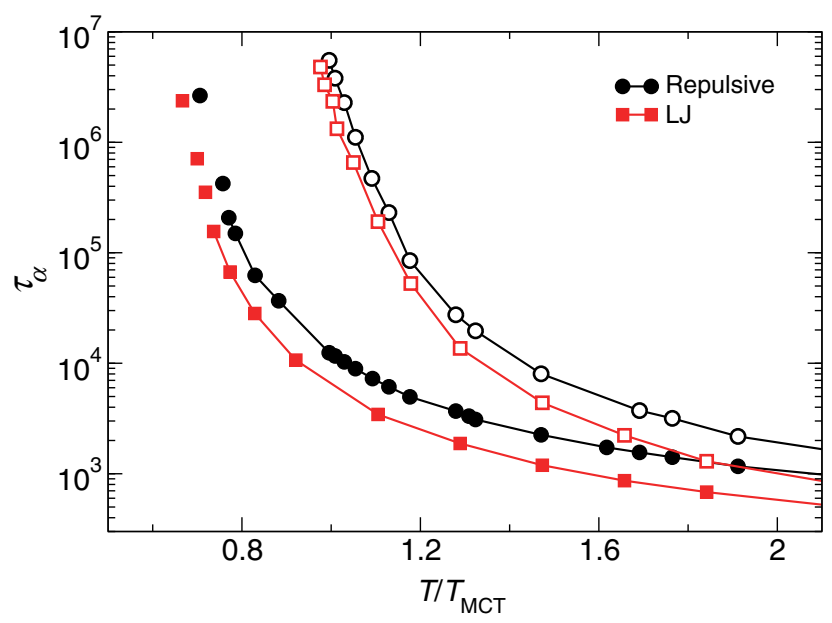

FIG. 15. Relaxation times for repulsive and Lennard-Jones potentials with a hybrid particle size distribution. Temperatures are scaled by $T_{\mathrm{MCT}}$ to allow direct comparison between models, with $T_{\mathrm{MCT}}=0.680$ and 0.543 for repulsive and LJ potentials, respectively. Open symbols represent the standard Monte Carlo dynamics, closed symbols the swap algorithm, for which unconnected symbols represent structurally unstable state points where only a rough estimate of $\tau_{\alpha}$ is obtained in short simulations.

stabilize the system down to even lower temperatures, but the main goal of this section has nevertheless been reached. We have indeed designed a model that is structurally similar to an equimolar binary mixture with size ratio 1.6 , but it can be efficiently studied using the swap algorithm down to $T=0.75 T_{\mathrm{MCT}}$ via the introduction of a third, intermediate specie. Using the same fitting procedure as above, we estimate that these low temperatures allow us to access a total dynamic range of about $\tau_{\alpha} / \tau_{0} \approx 10^{8}$, so the swap Monte Carlo method already allows the exploration of a novel temperature regime corresponding to an increase in relaxation times of about 3-4 orders of magnitude as compared to standard Monte Carlo simulations. It would be interesting to study hybrid variants of discrete mixtures, in which the concentration of specie $C$ is small enough to be considered as a small perturbation of the original model. Work in this direction is in progress.

\section{B. Lennard-Jones interactions}

Up to now, we have studied pair potentials describing repulsive soft spheres with inverse power-law repulsion of various softness. However, more realistic pair interactions including attractive forces are often used in studies of supercooled liquids. Perhaps the most studied pair interaction is the Lennard-Jones potential [58], which contains a soft power-law repulsion with exponent $n=12$, combined with a soft power-law attraction with exponent $n=6$ [see Eq. (7)].

In this final study, we test whether Lennard-Jones interactions can also be efficiently studied using the swap
Monte Carlo algorithm. To this end, we start from the previous "hybrid" model studied in Sec. VI A and include a power-law attraction. Because the potential is now longer-ranged, we use a larger cutoff $r_{\text {cut }}=2.5 \sigma_{i j}$ and shift the potential by the constant $c_{\mathrm{LJ}}$ to ensure the continuity of the potential at the cutoff. All other parameters are equal to the ones employed in the hybrid repulsive model in Sec. VI A.

We again perform a comparison of the standard and swap dynamics for this Lennard-Jones system and present the results along with the ones of the corresponding repulsive case in Fig. 15. We find that including attractive forces modifies both dynamics very little, apart from a rescaling of the temperature scale: The mode-coupling crossover temperature shifts from 0.680 to 0.543 when including attractive forces [84]. As a result, the above conclusions regarding stability and thermalization efficiency directly carry over to this Lennard-Jones system. Our main conclusion is therefore that our "feasibility study" is successful and that glass-forming models with Lennard-Jones interactions and a binarylike size distribution can be devised and studied down to very low temperatures using the swap algorithm. Such models will most certainly prove useful in future studies of the glass transition.

\section{PERSPECTIVES}

In this article, we established that a number of glassforming models with various pair interactions, particle size distributions, and degree of nonadditivity can be efficiently simulated using a simple swap Monte Carlo algorithm and remain excellent glass formers down to very low temperatures. For some models, we have been able to thermalize the metastable fluid down to temperatures that are lower than the laboratory glass transition, which represents the current experimental limit for molecular liquids. Therefore, our paper not only fills the 8-orders-of-magnitude gap between ordinary simulations and experimental work, but it actually goes beyond state-of-the-art experiments and demonstrates that both static and short-time dynamical properties can now be studied in computer simulations in a novel temperature regime. In addition to static quantities, by using thermalized configurations obtained with the swap method as initial conditions for trajectories generated without swap, we believe it is possible to substantially extend the dynamic window for structural relaxation, which may shed new light on the glassy dynamics as well.

Our achievements are summarized in Fig. 1, but throughout the article, we have suggested several ways in which our approach could be extended to devise different or more realistic models of glass-forming materials. We have also suggested ways in which the algorithm itself could be improved, and we have described several paths that remain to be explored in future work. We expect that these results will trigger much research activity towards these goals.

Obtaining thermalized states in simple models of supercooled liquids at temperatures comparable to the 
experimental glass transition paves the way to a number of novel studies because essentially all simulation work published over the past 30 years could be performed again over a previously inaccessible temperature regime. Some works along these lines have already been published [51,52], and others are currently in progress regarding the thermodynamic properties of deeply supercooled liquids, their local structure, vibrational and mechanical properties, and the existence of a Gardner transition in soft glasses.

\section{ACKNOWLEDGMENTS}

We thank G. Biroli, R. Jack, M. Ozawa, I. Procaccia, G. Tarjus, and M. Wyart for useful exchanges about this work. We thank R. Gutierrez for providing additional information regarding simulations performed in Ref. [50]. The research leading to these results has received funding from the European Research Council under the European Union Seventh Framework Programme (FP7/2007-2013)/ERC Grant Agreement No. 306845. This work was supported by a grant from the Simons Foundation (Grant No. 454933 to L. B.).

[1] L. Berthier and G. Biroli, Theoretical Perspective on the Glass Transition and Amorphous Materials, Rev. Mod. Phys. 83, 587 (2011).

[2] G. L. Hunter and E. R. Weeks, The Physics of the Colloidal Glass Transition, Rep. Prog. Phys. 75, 066501 (2012).

[3] M. D. Ediger, Spatially Heterogeneous Dynamics in Supercooled Liquids, Annu. Rev. Phys. Chem. 51, 99 (2000).

[4] G. Brambilla, D. El Masri, M. Pierno, L. Berthier, L. Cipelletti, G. Petekidis, and A. B. Schofield, Probing the Equilibrium Dynamics of Colloidal Hard Spheres Above the Mode-Coupling Glass Transition, Phys. Rev. Lett. 102, 085703 (2009).

[5] T. Blochowicz, A. Brodin, and E. A. Rössler, Evolution of the Dynamic Susceptibility in Supercooled Liquids and Glasses, in Fractals, Diffusion, and Relaxation in Disordered Complex Systems (Wiley-Blackwell, Hoboken, New Jersey, 2005), pp. 127-256.

[6] R. Richert and C. A. Angell, Dynamics of Glass-Forming Liquids. V. On the Link between Molecular Dynamics and Configurational Entropy, J. Chem. Phys. 108, 9016 (1998).

[7] P. G. Debenedetti and F. H. Stillinger, Supercooled Liquids and the Glass Transition, Nature (London) 410, 259 (2001).

[8] S. W. Hell, Far-Field Optical Nanoscopy, Science 316, 1153 (2007).

[9] S. Ashtekar, G. Scott, J. Lyding, and M. Gruebele, Direct Visualization of Two-State Dynamics on Metallic Glass Surfaces Well Below Tg, J. Phys. Chem. Lett. 1, 1941 (2010).

[10] S. F. Swallen, K. L. Kearns, M. K. Mapes, Y. S. Kim, R. J. McMahon, M. D. Ediger, T. Wu, L. Yu, and S. Satija, Organic Glasses with Exceptional Thermodynamic and Kinetic Stability, Science 315, 353 (2007).
[11] J.-L. Barrat, J.-N. Roux, and J.-P. Hansen, Diffusion, Viscosity and Structural Slowing Down in Soft Sphere Alloys Near the Kinetic Glass Transition, Chem. Phys. 149, 197 (1990).

[12] W. Kob and H.C. Andersen, Testing Mode-Coupling Theory for a Supercooled Binary Lennard-Jones Mixture. II. Intermediate Scattering Function and Dynamic Susceptibility, Phys. Rev. E 52, 4134 (1995).

[13] J. A. Anderson, C. D. Lorenz, and A. Travesset, General Purpose Molecular Dynamics Simulations Fully Implemented on Graphics Processing Units, J. Comp. Physiol. 227, 5342 (2008).

[14] P. H. Colberg and F. Höfling, Highly Accelerated Simulations of Glassy Dynamics Using GPUs: Caveats on Limited Floating-Point Precision, Comput. Phys. Commun. 182, 1120 (2011).

[15] N. P. Bailey, T. S. Ingebrigtsen, J. S. Hansen, A. A. Veldhorst, L. Bøhling, C. A. Lemarchand, A. E. Olsen, A. K. Bacher, H. Larsen, J. C. Dyre et al., RUMD: A General Purpose Molecular Dynamics Package Optimized to Utilize GPU Hardware Down to a Few Thousand Particles, arXiv:1506.05094.

[16] J. A. Anderson, E. Jankowski, T. L. Grubb, M. Engel, and S. C. Glotzer, Massively Parallel Monte Carlo for ManyParticle Simulations on GPUs, J. Comput. Phys. 254, 27 (2013).

[17] R. Meyer, Efficient Parallelization of Short-Range Molecular Dynamics Simulations on Many-Core Systems, Phys. Rev. E 88, 053309 (2013).

[18] M. E. J. Newman and G. T. Barkema, Monte Carlo Methods in Statistical Physics (Clarendon Press, Oxford, 1999).

[19] C. Dress and W. Krauth, Cluster Algorithm for Hard Spheres and Related Systems, J. Phys. A 28, L597 (1995).

[20] L. Santen and W. Krauth, Absence of Thermodynamic Phase Transition in a Model Glass Former, Nature (London) $\mathbf{4 0 5}$, 550 (2000).

[21] E. P. Bernard and W. Krauth, Two-Step Melting in Two Dimensions: First-Order Liquid-Hexatic Transition, Phys. Rev. Lett. 107, 155704 (2011).

[22] R. H. Swendsen and J.-S. Wang, Nonuniversal Critical Dynamics in Monte Carlo Simulations, Phys. Rev. Lett. 58, 86 (1987).

[23] K. Binder, The Monte-Carlo Methods in Condensed Matter Physics (Springer, Heidelberg, 1992).

[24] M. Isobe and W. Krauth, Hard-Sphere Melting and Crystallization with Event-Chain Monte Carlo, J. Chem. Phys. 143, 084509 (2015).

[25] R. L. C. Vink, A Finite-Temperature Monte Carlo Algorithm for Network Forming Materials, J. Chem. Phys. 140, 104509 (2014).

[26] K. Hukushima and K. Nemoto, Exchange Monte Carlo Method and Application to Spin Glass Simulations, J. Phys. Soc. Jpn. 65, 1604 (1996).

[27] K. Hukushima, H. Takayama, and H. Yoshino, Exchange Monte Carlo Dynamics in the SK Model, J. Phys. Soc. Jpn. 67, 12 (1998).

[28] R. Yamamoto and W. Kob, Replica-Exchange Molecular Dynamics Simulation for Supercooled Liquids, Phys. Rev. E 61, 5473 (2000). 
[29] G. Odriozola and L. Berthier, Equilibrium Equation of State of a Hard Sphere Binary Mixture at Very Large Densities Using Replica Exchange Monte Carlo Simulations, J. Chem. Phys. 134, 054504 (2011).

[30] W. Kob and L. Berthier, Probing a Liquid to Glass Transition in Equilibrium, Phys. Rev. Lett. 110, 245702 (2013).

[31] M. Ozawa, W. Kob, A. Ikeda, and K. Miyazaki, Equilibrium Phase Diagram of a Randomly Pinned Glass-Former, Proc. Natl. Acad. Sci. U.S.A. 112, 6914 (2015).

[32] L. Berthier, Overlap Fluctuations in Glass-Forming Liquids, Phys. Rev. E 88, 022313 (2013).

[33] L. Berthier and R. L. Jack, Evidence for a Disordered Critical Point in a Glass-Forming Liquid, Phys. Rev. Lett. 114, 205701 (2015).

[34] R. Faller and J. J. de Pablo, Density of States of a Binary Lennard-Jones Glass, J. Chem. Phys. 119, 4405 (2003).

[35] J. Callaham and J. Machta, Population Annealing Simulations of a Binary Hard Sphere Mixture, arXiv:1701.00263.

[36] D. Gazzillo and G. Pastore, Equation of State for Symmetric Non-additive Hard-Sphere Fluids: An Approximate Analytic Expression and New Monte Carlo Results, Chem. Phys. Lett. 159, 388 (1989).

[37] T. S. Grigera and G. Parisi, Fast Monte Carlo Algorithm for Supercooled Soft Spheres, Phys. Rev. E 63, 045102 (2001).

[38] G. Biroli, J.-P. Bouchaud, A. Cavagna, T. S. Grigera, and P. Verrocchio, Thermodynamic Signature of Growing Amorphous Order in Glass-Forming Liquids, Nat. Phys. 4, 771 (2008).

[39] C. Cammarota, A. Cavagna, G. Gradenigo, T. S. Grigera, and P. Verrocchio, Numerical Determination of the Exponents Controlling the Relationship between Time, Length, and Temperature in Glass-Forming Liquids, J. Chem. Phys. 131, 194901 (2009).

[40] C. Cammarota, A. Cavagna, I. Giardina, G. Gradenigo, T. S. Grigera, G. Parisi, and P. Verrocchio, Phase-Separation Perspective on Dynamic Heterogeneities in Glass-Forming Liquids, Phys. Rev. Lett. 105, 055703 (2010).

[41] A. Cavagna, T. S. Grigera, and P. Verrocchio, Dynamic Relaxation of a Liquid Cavity under Amorphous Boundary Conditions, J. Chem. Phys. 136, 204502 (2012).

[42] S. Pronk and D. Frenkel, Melting of Polydisperse Hard Disks, Phys. Rev. E 69, 066123 (2004).

[43] L. A. Fernández, V. Martín-Mayor, and P. Verrocchio, Phase Diagram of a Polydisperse Soft-Spheres Model for Liquids and Colloids, Phys. Rev. Lett. 98, 085702 (2007).

[44] L. A. Fernández, V. Martín-Mayor, B. Seoane, and P. Verrocchio, Separation and Fractionation of Order and Disorder in Highly Polydisperse Systems, Phys. Rev. E 82, 021501 (2010).

[45] B. Bernu, J. P. Hansen, Y. Hiwatari, and G. Pastore, SoftSphere Model for the Glass Transition in Binary Alloys: Pair Structure and Self-Diffusion, Phys. Rev. A 36, 4891 (1987).

[46] L. A. Fernández, V. Martín-Mayor, and P. Verrocchio, Critical Behavior of the Specific Heat in Glass Formers, Phys. Rev. E 73, 020501 (2006).

[47] Y. Brumer and D. R. Reichman, Numerical Investigation of the Entropy Crisis in Model Glass Formers, J. Phys. Chem. B 108, 6832 (2004).
[48] L. A. Fernández, V. Martín-Mayor, and P. Verrocchio, Optimized Monte Carlo Method for Glasses, Philos. Mag. 87, 581 (2007).

[49] L. Santen and W. Krauth, Liquid, Glass and Crystal in TwoDimensional Hard Disks, arXiv:cond-mat/0107459.

[50] R. Gutiérrez, S. Karmakar, Y. G. Pollack, and I. Procaccia, The Static Lengthscale Characterizing the Glass Transition at Lower Temperatures, Europhys. Lett. 111, 56009 (2015).

[51] L. Berthier, D. Coslovich, A. Ninarello, and M. Ozawa, Equilibrium Sampling of Hard Spheres up to the Jamming Density and Beyond, Phys. Rev. Lett. 116, 238002 (2016).

[52] L. Berthier, P. Charbonneau, Y. Jin, G. Parisi, B. Seoane, and F. Zamponi, Growing Timescales and Lengthscales Characterizing Vibrations of Amorphous Solids, Proc. Natl. Acad. Sci. U.S.A. 113, 8397 (2016).

[53] S. Sastry, P. G. Debenedetti, and F. H. Stillinger, Signatures of Distinct Dynamical Regimes in the Energy Landscape of a Glass-Forming Liquid, Nature (London) 393, 554 (1998).

[54] M. P. Allen and D. J. Tildesley, Computer Simulation of Liquids (Clarendon Press, Oxford, England, 1989).

[55] D. Frenkel and B. Smit, Understanding Molecular Simulation, 2nd ed. (Academic, New York, 2001).

[56] L. Berthier and W. Kob, The Monte Carlo Dynamics of a Binary Lennard-Jones Glass-Forming Mixture, J. Phys. Condens. Matter 19, 205130 (2007).

[57] K. Zhang, M. Fan, Y. Liu, J. Schroers, M. D. Shattuck, and C. S. O'Hern, Beyond Packing of Hard Spheres: The Effects of Core Softness, Non-additivity, Intermediate-Range Repulsion, and Many-Body Interactions on the Glass-Forming Ability of Bulk Metallic Glasses, J. Chem. Phys. 143, 184502 (2015).

[58] W. Kob and H.C. Andersen, Scaling Behavior in the Beta-Relaxation Regime of a Supercooled Lennard-Jones Mixture, Phys. Rev. Lett. 73, 1376 (1994).

[59] Ch. Hausleitner and J. Hafner, Hybridized Nearly-FreeElectron Tight-Binding-Bond Approach to Interatomic Forces in Disordered Transition-Metal Alloys. I. Theory, Phys. Rev. B 45, 115 (1992).

[60] J. P. Hansen and I. R. McDonald, Theory of Simple Liquids: With Applications to Soft Matter (Academic Press, Amsterdam, Boston, 2013).

[61] H. Tanaka, Bond Orientational Order in Liquids: Towards a Unified Description of Water-like Anomalies, Liquid-Liquid Transition, Glass Transition, and Crystallization, Eur. Phys. J. E 35113 (2012).

[62] W. Götze, Complex Dynamics of Glass-Forming Liquids: A Mode-Coupling Theory (Oxford University Press, Oxford, New York, 2009).

[63] Y. S. Elmatad, D. Chandler, and J. P. Garrahan, Corresponding States of Structural Glass Formers, J. Phys. Chem. B 113, 5563 (2009).

[64] P. Sollich and N. B. Wilding, Phase Behaviour of Polydisperse Spheres: Simulation Strategies and an Application to the Freezing Transition, J. Chem. Phys. 133, 224102 (2010).

[65] P. Sollich and N. B. Wilding, Crystalline Phases of Polydisperse Spheres, Phys. Rev. Lett. 104, 118302 (2010).

[66] T. S. Ingebrigtsen and H. Tanaka, Effect of Size Polydispersity on the Nature of Lennard-Jones Liquids, J. Phys. Chem. B 119, 11052 (2015). 
[67] D. A. Mártin, A. Cavagna, and T. S. Grigera, Specific Heat Anomaly in a Supercooled Liquid with Amorphous Boundary Conditions, Phys. Rev. Lett. 114, 225901 (2015).

[68] B. Bernu, J. P. Hansen, Y. Hiwatari, and G. Pastore, SoftSphere Model for the Glass Transition in Binary Alloys: Pair Structure and Self-Diffusion, Phys. Rev. A 36, 4891 (1987).

[69] L. Berthier, G. Biroli, D. Coslovich, W. Kob, and C. Toninelli, Finite-Size Effects in the Dynamics of GlassForming Liquids, Phys. Rev. E 86031502 (2012).

[70] K. Zhang, B. Dice, Y. Liu, J. Schroers, M. D. Shattuck, and C. S. O'Hern, On the Origin of Multi-Component Bulk Metallic Glasses: Atomic Size Mismatches and De-mixing, J. Chem. Phys. 143, 054501 (2015).

[71] C. De Michele, F. Sciortino, and A. Coniglio, Scaling in Soft Spheres: Fragility Invariance on the Repulsive Potential Softness, J. Phys. Condens. Matter 16, L489 (2004).

[72] I. Douglass, T. Hudson, and P. Harrowell, Density and Glass Forming Ability in Amorphous Atomic Alloys: The Role of the Particle Softness, J. Chem. Phys. 144, 144502 (2016).

[73] D. Gazzillo, G. Pastore, and S. Enzo, Chemical Short-Range Order in Amorphous Ni-Ti Alloys: An Integral Equation Approach with a Non-additive Hard-Sphere Model, J. Phys. Condens. Matter 1, 3469 (1989).

[74] D. Gazzillo, G. Pastore, and R. Frattini, The Role of Excluded Volume Effects on the Structure and Chemical Short-Range Order of $\mathrm{Ni}_{33} \mathrm{Y}_{67}$ Metallic Glass, J. Phys. Condens. Matter 2, 8463 (1990).

[75] U. Buchenau and R. Zorn, A Relation between Fast and Slow Motions in Glassy and Liquid Selenium, Europhys. Lett. 18, 523 (1992).
[76] T. Scopigno, G. Ruocco, F. Sette, and G. Monaco, Is the Fragility of a Liquid Embedded in the Properties of Its Glass?, Science 302, 849 (2003).

[77] J. C. Dyre, Colloquium: The Glass Transition and Elastic Models of Glass-Forming Liquids, Rev. Mod. Phys. 78, 953 (2006).

[78] H. W. Hansen, B. Frick, T. Hecksher, J. C. Dyre, and K. Niss, Connection between Fragility, Mean-Squared Displacement, and Shear Modulus in Two van der Waals Bonded GlassForming Liquids, Phys. Rev. B 95, 104202 (2017).

[79] C. Toninelli, M. Wyart, L. Berthier, G. Biroli, and J.-P. Bouchaud, Dynamical Susceptibility of Glass Formers: Contrasting the Predictions of Theoretical Scenarios, Phys. Rev. E 71, 041505 (2005).

[80] L. Berthier, G. Biroli, J.-P. Bouchaud, W. Kob, K. Miyazaki, and D. R. Reichman, Spontaneous and Induced Dynamic Fluctuations in Glass Formers. I. General Results and Dependence on Ensemble and Dynamics, J. Chem. Phys. 126, 184503 (2007).

[81] L. Berthier and R. L. Jack, Structure and Dynamics of Glass Formers: Predictability at Large Length Scales, Phys. Rev. E 76, 041509 (2007).

[82] R. L. Jack, A. J. Dunleavy, and C. P. Royall, InformationTheoretic Measurements of Coupling between Structure and Dynamics in Glass Formers, Phys. Rev. Lett. 113, 095703 (2014).

[83] G. Wahnström, Molecular-Dynamics Study of a Supercooled Two-Component Lennard-Jones System, Phys. Rev. A 44, 3752 (1991).

[84] L. Berthier and Gilles Tarjus, The Role of Attractive Forces in Viscous Liquids, J. Chem. Phys. 134, 214503 (2011). 